\title{
Structure and Dynamics of Self-assembled Comb Copolymers: Comparison between Simulations of a Generic Model and Neutron Scattering Experiments
}

\author{
Angel J. Moreno, ${ }^{1, *}$ Arantxa Arbe, ${ }^{1}$ and Juan Colmenero ${ }^{1,2,3}$ \\ ${ }^{1}$ Centro de Física de Materiales (CSIC, \\ UPV/EHU) and Materials Physics Center MPC, \\ Paseo Manuel de Lardizabal 5, E-20018 San Sebastián, Spain. \\ ${ }^{2}$ Donostia International Physics Center, \\ Paseo Manuel de Lardizabal 4, E-20018 San Sebastián, Spain. \\ ${ }^{3}$ Departamento de Física de Materiales, \\ Universidad del País Vasco (UPV/EHU), \\ Apartado 1072, E-20080 San Sebastián, Spain.
}

We have performed extensive molecular dynamic simulations on a simple beadspring model for block copolymers with comb-like architecture. Monomers located at the main chain and at the arms are respectively denoted as S ('slow') and F ('fast'). The model parameters are selected in order to induce segregation in domains rich in one component and poor in the other. In particular we investigate the case in which the linear homopolymer of F-monomers exhibits much faster intrinsic dynamics than the S-counterpart. As a consequence, a strong dynamic asymmetry between both components is still present in the self-assembled copolymer system. We investigate static and dynamic properties as a function of arm length and temperature. The fast component exhibits decoupling of self- and collective dynamics, as well as strongly stretched relaxation. Stretching is an intrinsic feature and is not necessarily related to gradients of mobility. The observed qualitative trends are fully consistent with recent neutron scattering experiments on poly( $n$-alkyl methacrylates), suggesting that they are generic in comb copolymers with strong dynamic asymmetry.

\footnotetext{
${ }^{*}$ Corresponding author: wabmosea@ehu.es
} 


\section{INTRODUCTION}

During the last decades the structure and dynamics of comb-like polymers have been extensively studied by different experimental techniques. In most of the investigated systems the side-group ('arm') contains an alkyl chain of varying length, and the main-chain backbone is much more rigid. This is the case of the families of poly $(n$-alkyl methacrylates) (PnMAs) [1-22], poly(n-alkyl acrylates) $[2,16,23]$, poly(di- $n$-alkyl itaconates) [2426], poly(3-alkyl thiophenes) (P3ATs) [27, 28], rod polyimides [29], polyazomethynes [30], poly(p-phenylene-terephthalates) [31, 32], or poly( $\alpha$ - $n$-alkyl $\beta$-L-aspartates) [33]. Other comb copolymers with polar conformationally versatile arms including phenyl rings have also been investigated [34]. Some of the above mentioned systems show some degree of crystallinity, and their structural and dynamical properties naturally differ from those characteristic for amorphous main-chain polymers or amorphous polymers with small side-groups. However, even in absence of crystallinity, some structural and dynamical peculiarities emerge in comb-like polymers. From X-ray experiments, a kind of nanophase separation has been suggested in the systems with alkyl side groups: arm- and main-chain units tend to aggregate separately, forming self-assembled nanodomains [12, 16, 25]. Apparently, this nanophase separation also occurs in comb copolymers with chemically more complex arms [34]. Concerning dynamic properties, the presence of long or bulky arms has also a strong impact on the relaxation processes of polymer chains. On the one hand, a strong plasticization for the main-chain dynamics is observed. This effect, discussed already in the 1960's [1] is enhanced by increasing the arm length, leading to important shifts in the associated glass-transition temperature [21, 22]. On the other hand, mainly based on calorimetric, mechanical and dielectric spectroscopy studies, the appearance of an independent dynamics within the side-group nanodomains has been suggested for polymers with long alkyl side-chains $[9,16,17,27]$. Interestingly enough, this process seems to be independent of the packing of the main chains, since similar associated relaxation spectra are observed in the case of P3ATs with either crystalline or amorphous main-chains [28]. Employing complementary NMR techniques -that are selective for the different molecular groups in the system- further evidences for the scenario of independent main-chain and side-group dynamics were brought forward [17]. The need of techniques selective at molecular level for the investigation of the structure and the dynamics of this kind of systems turns to be 
clearly imperative.

Recently, neutron scattering combined with isotopic labelling was employed to selectively study the two components (main chain and arms) in some PnMAs [21, 22]. The neutron diffraction experiments provided strong support to the existence of a nanosegregated structure (note that in X-ray diffraction mostly carbons and oxygens are highlighted, without the possibility of distinguishing main-chain and arm contributions to the diffraction patterns). Moreover, while the structural relaxation of the main chains showed standard features, anomalous behavior emerged for the dynamics of the alkyl units in systems with long arms. This includes extremely stretched functional forms, Arrhenius-like behavior and decoupling of self- and collective motions [21,22]. To rationalize these observations two main possible ingredients were invoked: distributions of relaxation times along the arms and confinement by the much slower relaxation of the rigid main-chain matrix. In this framework, the dynamic asymmetry between the two subsystems would play an essential role. Several questions remain unsolved, namely: is nanosegregation induced by incompatibility of main chains and arms, as usually assumed [16], or can it be entropically driven by the comb-like architecture of the polymer? Does the main-chain component show 'standard' behavior also at length scales smaller than the average inter-molecular distances? Are the distributions of mobilities within the arms as broad as those obtained by a phenomenological analysis of the experimental data? [22]

In this work we aim to shed light on these questions by means of molecular dynamics simulations. These provide a direct calculation, from the atomic trajectories, of observables that cannot be accessed in experiments or that are indirectly obtained from them on the basis of approximations or model assumptions. The main limitations of simulation techniques are given by the accuracy of the used force field and by the computational resources. Even by parallel computation in standard modern machines, it is demanding to simulate polymer melts with fully atomistic force fields for, e.g., time scales of 100 nanoseconds and cell dimensions of $50 \AA$. This constitutes a clear limitation for the case of nanostructured phases, as those investigated in this work, and in particular for a systematic investigation of the structure and dynamics by varying several control parameters (e.g., temperature and arm length).

Simulations of bead-spring models of macromolecules allow to access much larger time and length scales, at the expense of substituting the chemical structure by simple chain 
backbones, and reducing the force field to a few contributions describing the basic ingredients of the interactions (e.g., excluded volume and connectivity). Despite its simplicity they usually provide an excellent description of qualitative structural and dynamic features of polymer-based systems in very different and complex physical situations [35-37]. Due the generic character of the interactions, bead-spring models are also very useful for tests of theories and predictions of universal properties present in polymer systems of very different chemical composition.

With these ideas in mind we have performed extensive simulations of a bead-spring model for comb copolymers with dynamic asymmetry, namely with the slow and fast components located respectively at the main chain and at the arms. We investigate structural and dynamic properties for a broad range of temperature and arm length. The static and dynamic correlators obtained from simulations reproduce the trends observed in experiments on $\operatorname{poly}(n$-alkyl methacrylates) $[21,22]$. We confirm the proposed scenario of nanodomain formation. Moreover we show that the latter can have a purely entropic origin, and takes place even for homopolymer combs provided that the density of branch points is high enough. It is found that the dynamics of the main-chain monomers is standard at all length scales. In analogy with the experiments, the analysis of scattering functions reveals a decoupling of self- and collective dynamics for the fast component in the arms, and strongly stretched relaxation. Characterization of dynamic heterogeneity shows that stretching is an intrinsic feature and is not necessarily related to gradients of mobility along the arms. Due to the generic character of the bead-spring model, we suggest that the observed scenario will be a general feature of comb copolymers with dynamic asymmetry.

The paper is organized as follows. Model and simulation details are given in Section II. Structural and dynamic features are discussed respectively in Sections III and IV, in comparison with experimental results in poly(n-alkyl methacrylates). Further details of the experimental part can be found in the recent publication [22] and references therein. Conclusions are given in Section V.

\section{MODEL AND SIMULATIONS DETAILS}

We have simulated melts of identical bead-spring macromolecules with comb-like architecture (see Figure 1). Monomers located at the main chain and at the arms are respectively 


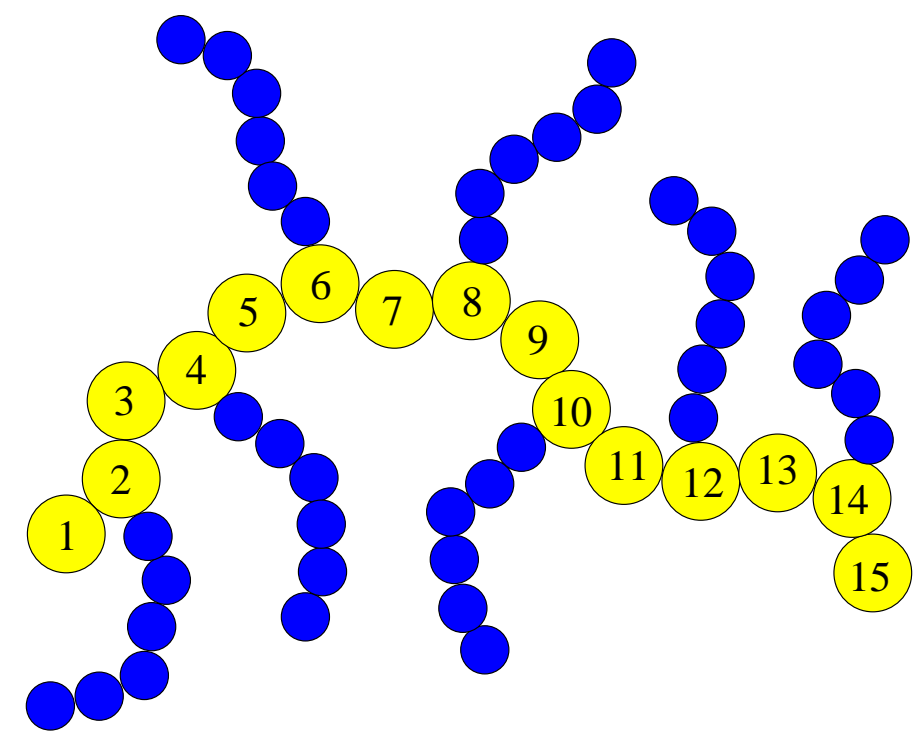

FIG. 1: Architecture of the simulated SF-combs, for the case $N_{\mathrm{F}}=6$. Light and dark spheres correspond respectively to S- and F-monomers.

denoted as S ('slow') and F ('fast'). For simplicity both type of monomers have identical mass $m=1$. The main chain consists of $N_{\mathrm{S}}=15$ S-monomers, and $N_{\text {arm }}=7$ identical F-arms regularly linked to the main chain. Specifically, by denoting the S-monomers as $i=1,2, \ldots, 15$ from one end of the main chain to the other one (Figure 1), the F-arms are linked to $i=2,4,6,8,10,12$, and 14. Each arm consists of $N_{\mathrm{F}}$ F-monomers. We simulate systems with $N_{\mathrm{F}}=2,3,4,5$, and 6 . This corresponds to number fractions of F-monomers, $x_{\mathrm{F}}$, ranging from 0.48 to 0.74 . We have also simulated pure S-combs. These have the same architecture as the respective S-F combs, i.e., each F-monomer in the arms is just substituted by an S-monomer. Additional simulations have been performed for pure linear Sand F-homopolymers, with chains of 15 monomers in both cases. Details of the size of the investigated systems are given in Table I.

The non-bonded interaction between any two given monomers of the species $\alpha$ and $\beta$ $\in\{\mathrm{S}, \mathrm{F}\}$ is given by the Weeks-Chandler-Andersen (WCA) potential [38]:

$$
V_{\alpha \beta}(r)=4 \epsilon_{\alpha \beta}\left[\left(\frac{\sigma_{\alpha \beta}}{r}\right)^{12}-\left(\frac{\sigma_{\alpha \beta}}{r}\right)^{6}+\frac{1}{4}\right],
$$

for $r<r_{\mathrm{c}}$ and $V_{\alpha \beta}(r)=0$ for $r \geq r_{\mathrm{c}}$. By using a value $r_{\mathrm{c}}=2^{1 / 6} \sigma_{\alpha \beta}$, potential and forces are continuous at the cutoff distance. The WCA potential is purely repulsive and has no local minima, aiming to mimic excluded volume effects. Two bonded monomers in a same chain 


\begin{tabular}{ccccc}
$N_{\mathrm{F}}, N_{\mathrm{a}}$ & Formula & $x_{\mathrm{F}}$ & $N_{\text {mac }}$ & $N_{\text {mon }}$ \\
\hline & & & & \\
2 & $\mathrm{~S}_{15} \mathrm{~F}_{14}$ & 0.48 & 1000 & 29000 \\
3 & $\mathrm{~S}_{15} \mathrm{~F}_{21}$ & 0.58 & 1000 & 36000 \\
4 & $\mathrm{~S}_{15} \mathrm{~F}_{28}$ & 0.65 & 900 & 38700 \\
5 & $\mathrm{~S}_{15} \mathrm{~F}_{35}$ & 0.70 & 800 & 40000 \\
6 & $\mathrm{~S}_{15} \mathrm{~F}_{42}$ & 0.74 & 700 & 39900 \\
2 & $\mathrm{~S}_{15} \mathrm{~S}_{14}$ & & 600 & 17400 \\
3 & $\mathrm{~S}_{15} \mathrm{~S}_{21}$ & & 500 & 18000 \\
4 & $\mathrm{~S}_{15} \mathrm{~S}_{28}$ & & 400 & 17200 \\
5 & $\mathrm{~S}_{15} \mathrm{~S}_{35}$ & & 300 & 15000 \\
6 & $\mathrm{~S}_{15} \mathrm{~S}_{42}$ & & 300 & 17100 \\
& $\mathrm{~S}_{15}$ & & 1206 & 18090 \\
& $\mathrm{~F}_{15}$ & & 1200 & 18000
\end{tabular}

TABLE I: Details of the size of the simulated systems. $N_{\mathrm{F}}$ : number of F-monomers per arm in SF-combs. $N_{\mathrm{a}}$ : number of monomers per arm in pure S-combs. $x_{\mathrm{F}}$ : fraction of F-monomers in SF-combs. $N_{\text {mac }}$ and $N_{\text {mon }}$ : respectively, number of macromolecules and monomers in the simulation box. Formulae $\mathrm{S}_{15} \mathrm{~F}_{a}$ denote $\mathrm{SF}$-combs, with 15 and $a$ the number of, respectively, Sand F-monomers per comb. Formulae $\mathrm{S}_{15} \mathrm{~S}_{a}$ denote pure S-combs, with 15 and $a$ the number of monomers per comb in, respectively, the main chain and the arms. Formulae $S_{15}$ and $F_{15}$ denote respectively linear S- and F-homopolymers, with 15 monomers per chain.

also interact through a finitely extensible nonlinear elastic potential (FENE) [39]:

$$
V_{\alpha \beta}^{\mathrm{FENE}}(r)=-\epsilon_{\alpha \beta} K_{\mathrm{F}} R_{0}^{2} \ln \left[1-\left(\frac{r}{R_{0} \sigma_{\alpha \beta}}\right)^{2}\right],
$$

with $K_{\mathrm{F}}=15$ and $R_{0}=1.5$. The sum of eqs 1 and 2 yields an effective potential between bonded monomers with a sharp minimum at $r=0.96 \sigma_{\alpha \beta}$ and guarantees chain uncrossability [39]. We use identical energy scales $\epsilon_{\alpha \beta}=1$ and distinct interaction diameters $\sigma_{\mathrm{SS}}=1.6$, $\sigma_{\mathrm{FF}}=1.0$, and $\sigma_{\mathrm{SF}}=1.3$. With these selected values, at a fixed temperature $T$ and pressure 
$P$, the F-homopolymer exhibits much faster dynamics than the S-homopolymer and dynamic asymmetry arises in the SF-comb system (see below).

In the following, temperature $T$, number density $\rho$, pressure $P$, time $t$, distance, and wave vector $q$ will be given respectively in units of $\epsilon / k_{\mathrm{B}}$ (with $k_{\mathrm{B}}$ the Boltzmann constant), $\sigma_{\mathrm{FF}}^{-3}, \epsilon \sigma_{\mathrm{FF}}^{-3}, \sigma_{\mathrm{FF}}(m / \epsilon)^{1 / 2}, \sigma_{\mathrm{FF}}$, and $\sigma_{\mathrm{FF}}^{-1}$. Cubic periodic boundary conditions are implemented. Computation time is reduced by using a linked-cell method [40]. The simulations are performed in the isothermal-isobaric (NPT) ensemble, by using the Nosé-Hoover algorithm [40] at external pressure $P=2.5$. Equations of motion are integrated in the Martyna's scheme $[40,41]$, with a time step ranging from $\delta t=10^{-4}$ to $3 \times 10^{-3}$, depending on the simulated temperature.

The system is prepared by placing and growing the chains randomly at a low density, with a constraint that avoids initial overlap of monomers within distances $r<0.8 \sigma_{\alpha \beta}$. We start an NPT equilibration run with applied external pressure $P=2.5$. The system is considered to be equilibrated when no drift is observed for the volume of the simulation box and the thermodynamic quantities (internal pressure, kinetic and potential energy). Likewise no aging effects are observed for static and dynamic correlators. Then we start a new NPT run, at the same $P=2.5$, for production of configurations, from which we compute static and dynamic correlators. Typical simulation times of both equilibration and production runs are of 30 million time steps. Static correlators are averaged over typically 40 equispaced configurations. Dynamic correlators are averaged over 20 equispaced time origins.

We define the effective packing fraction as $\Phi=\left[\pi /\left(6 L_{\text {box }}^{3}\right)\right]\left[N_{\mathrm{mon}}^{\mathrm{S}} \sigma_{\mathrm{SS}}^{3}+N_{\mathrm{mon}}^{\mathrm{F}} \sigma_{\mathrm{FF}}^{3}\right]$, with $N_{\text {mon }}^{\alpha}$ the total number of $\alpha$-monomers in the simulation box, of size $L_{\text {box }}$. Since all the simulations are done at fixed $P=2.5, \Phi$ depends on temperature. For temperatures of interest we find typical values of $\Phi$ ranging from 0.52 to 0.57 , which are characteristic of melt conditions in bead-spring models of polymer systems [35-37, 39]. Finally, we note that a direct mapping of the parameters corresponding to the simulated and the real systems is not possible, preventing quantitative comparisons of the results on both kinds of systems. 


\section{STRUCTURE}

Studies by X-ray diffraction suggest nanophase formation in comb-like copolymers. This hypothesis is based on the existence of a prepeak at low $q$-values, at a position $q_{\text {I }}$ that shifts towards lower $q$-values with increasing arm length. This prepeak is also present in the neutron diffraction results on fully deuterated PnMAs [21, 22] and exhibits the same trend, as shown in the inset of Figure 2. There, data corresponding to polymers with different number, $n_{\mathrm{C}}$, of alkyl C-atoms in the arm are compared $\left(n_{\mathrm{C}}=2,4\right.$ and 6$)$. The coherent cross-section $(\partial \sigma / \partial \Omega)_{\text {coh }}$ accessed in neutron scattering experiments is given by [42] $(\partial \sigma / \partial \Omega)_{\mathrm{coh}}=\sum_{\alpha \beta} \bar{b}_{\alpha} \bar{b}_{\beta} S_{\alpha \beta}(q)$, where the partial static structure factors involving the isotopes $\alpha$ and $\beta$ are defined as

$$
S_{\alpha \beta}(q)=\frac{\left\langle\rho_{\mathbf{q}}^{\alpha}(0) \rho_{-\mathbf{q}}^{\beta}(0)\right\rangle}{\sqrt{N^{\alpha} N^{\beta}}},
$$

with $\rho_{\mathbf{q}}^{\alpha}(t)=\sum_{j=1}^{N^{\alpha}} \exp \left[i \mathbf{q} \cdot \mathbf{r}_{j}(t)\right]$. Here $\mathbf{r}_{j}$ denotes the atomic coordinates for the atom $j$ of the species $\alpha$, from which there are a total of $N^{\alpha}$ atoms in the sample. The brackets denote thermal and orientational (over the directions of $\mathbf{q}$ ) average.

The contribution of a given pair correlation involving isotopes $\alpha, \beta$ to the coherently scattered intensity is weighted by the corresponding scattering lengths $\bar{b}_{\alpha}$ and $\bar{b}_{\beta}$ (see above). In fully deuterated samples all the atomic pair correlations are almost identically weighted $\left(\bar{b}_{\mathrm{C}}=6.6511 \mathrm{fm} \simeq \bar{b}_{\mathrm{D}}=6.671 \mathrm{fm}\right.$, and $\left.\bar{b}_{\mathrm{O}}=5.803 \mathrm{fm}\right)$. Thus, the coherently scattered intensity provides a good approximation of the total static structure factor,

$$
S(q)=\left\langle\rho_{\mathbf{q}}(0) \rho_{-\mathbf{q}}(0)\right\rangle / N_{\text {at }},
$$

with $\rho_{\mathbf{q}}(t)=\rho_{\mathbf{q}}^{\alpha}(t)+\rho_{\mathbf{q}}^{\beta}(t)$, and $N_{\text {at }}=N^{\alpha}+N^{\beta}$ the total number of atoms in the sample. Neutron diffraction with polarization analysis has been performed on partially deuterated samples where the arm is protonated and the main chain is deuterated. Since $\bar{b}_{\mathrm{H}}=-3.7406$ $\mathrm{fm}$ and $\bar{b}_{\mathrm{C}}=6.6511 \mathrm{fm}$, the scattering length density of the methylene $\left(-\mathrm{CH}_{2}-\right)$ groups in the arms is negligible. Thus, with this partial deuteration the arms become practically invisible for neutrons [42]. Figure 2 shows the measured coherent cross-section for poly(hexyl methacrylate), PHMA $\left(n_{\mathrm{C}}=6\right)$, on a fully deuterated and a partially deuterated sample in absolute units. The vanishment of the second peak at $q_{\mathrm{II}} \sim 1.3 \AA^{-1}$ in the protonated-arm sample demonstrates that this peak reflects pair correlations involving atoms in the arms. 


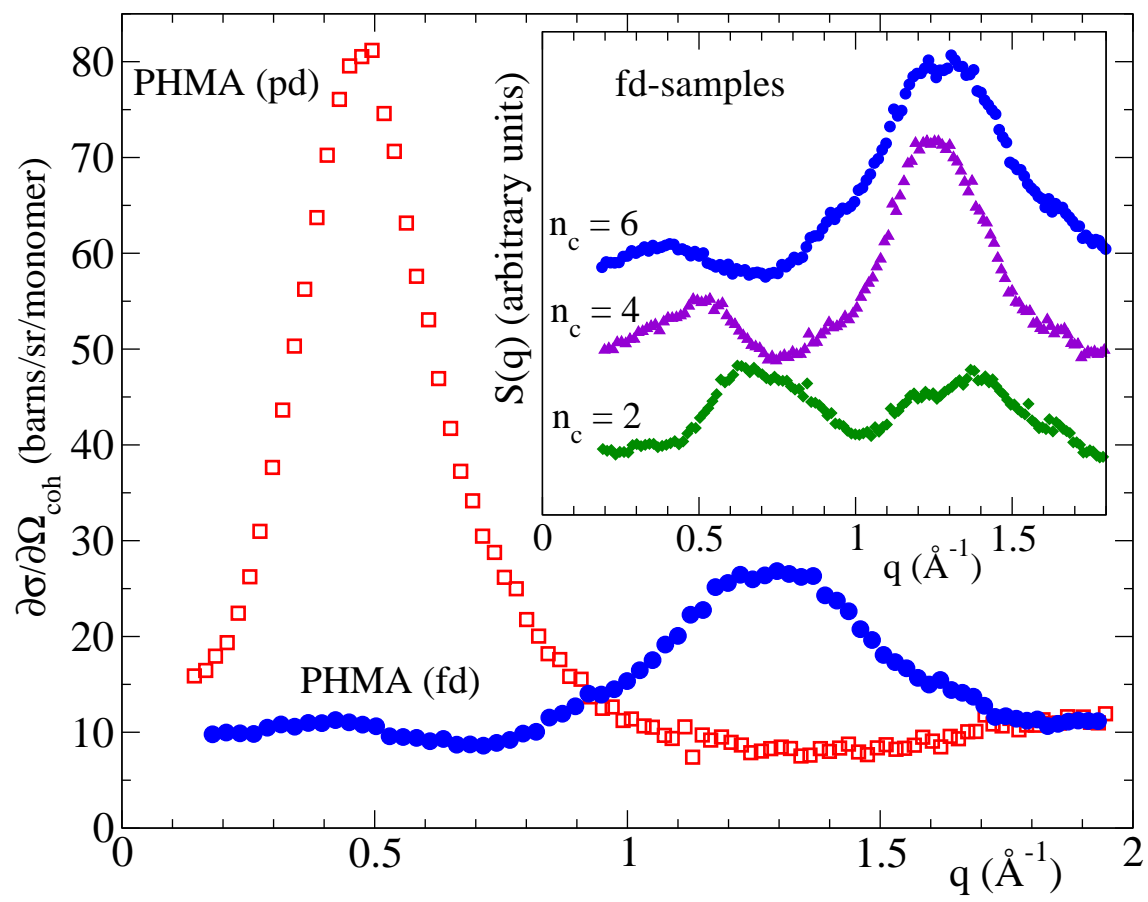

FIG. 2: Main panel: coherent scattering cross-section measured on fully deuterated (fd, full circles) and partially deuterated ( $\mathrm{pd}$, empty squares) samples of PHMA $\left(n_{\mathrm{C}}=6\right)$. The inset shows the static structure factors measured on the fully deuterated samples with $n_{\mathrm{C}}=6,4$ and 2 from top to bottom. The different data sets in the inset are plotted in arbitrary units, and relative shifts have been applied for clarity. Temperatures for each sample are about $75 \mathrm{~K}$ above the respective calorimetric glass transition temperatures [21].

Moreover, its similarity with the intermolecular diffraction peak in molten polyethylene (PE) [21] points to a PE-like environment for the arm units, supporting the proposed scenario of alkyl nanodomains. On the other hand, the prepeak at $0.3 \AA^{-1} \lesssim q_{\mathrm{I}} \lesssim 0.7 \AA^{-1}$ remains in the partially deuterated sample and therefore reflects pair correlations between atoms in the main chain. On this basis, the origin of this prepeak has been attributed to correlations between main chains delimiting the alkyl nanodomains [21, 22].

The former structural scenario is confirmed by the simulations. Figure 3 shows a typical snapshot of the simulation box for SF-combs with arm length $N_{\mathrm{F}}=6$. This provides direct visual evidence of the formation of the nanodomains. It must be noted that the starting configuration of the system is random (see above). Therefore, the domains are formed spontaneously. The panel (a) of Figure 4 shows the total static structure factor 


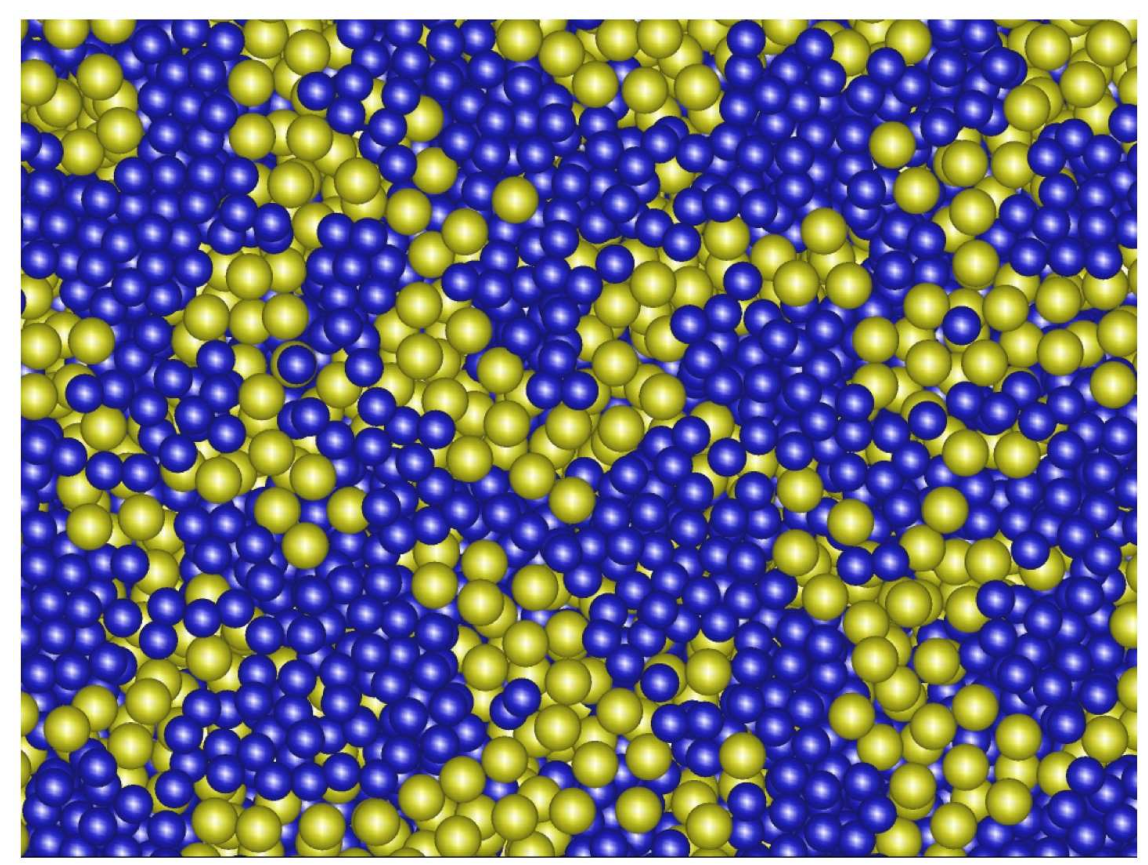

FIG. 3: Partial snapshot of a typical configuration of the SF-system, with $N_{\mathrm{F}}=6$. Light and dark spheres corresponds respectively to S- and F-monomers.

$S(q)$. The panels (b)-(d) display the different partial contributions $S_{\alpha \beta}(q)$. The former quantities are defined as above [43] (Equations 3 and 4), with the 'isotopes' $\alpha \in\{\mathrm{S}, \mathrm{F}\}$. Both total and partial structure factors exhibit a first peak, $q_{\mathrm{I}}$, in the range $1.1 \lesssim q_{\mathrm{I}} \lesssim 1.9$. It shifts to lower $q$, i.e., to larger length scales, on increasing the arm length. The peak is negative in the case of $S_{\mathrm{SF}}$, revealing a strong anticorrelation between both species in the associated length scale $2 \pi / q_{\mathrm{I}}$ (of $\approx 3 \sigma_{\mathrm{BB}}$ for $N_{\mathrm{F}}=2$ and $\approx 6 \sigma_{\mathrm{BB}}$ for $N_{\mathrm{F}}=6$ ). The former features around $q_{\text {I }}$ just reflect the formation of the nanodomains, which increase their size on increasing the arm length. The observed shift of the peak to lower $q$ for longer arms is in agreement with the experimental observation (see inset of Figure 2). Thus, the prepeak at $q_{\mathrm{I}}$ is characteristic for inter-domain correlations, both reflected by main-chain/main-chain or by arm/arm correlations. [?]

At this point, we make a comment on the interpretation of the experiments in Ref. [21]. Simulation results of Figures 3 and 4 show that the interpretation in terms of nanodomain formation is correct, but the assignment of $q_{\mathrm{I}}$ to only correlations between main-chain units has to be clarified. As shown in Figure $4, q_{\text {I }}$ does characterize correlations between mainchain units, but also correlations between arm units and cross-correlations. In fact, the 

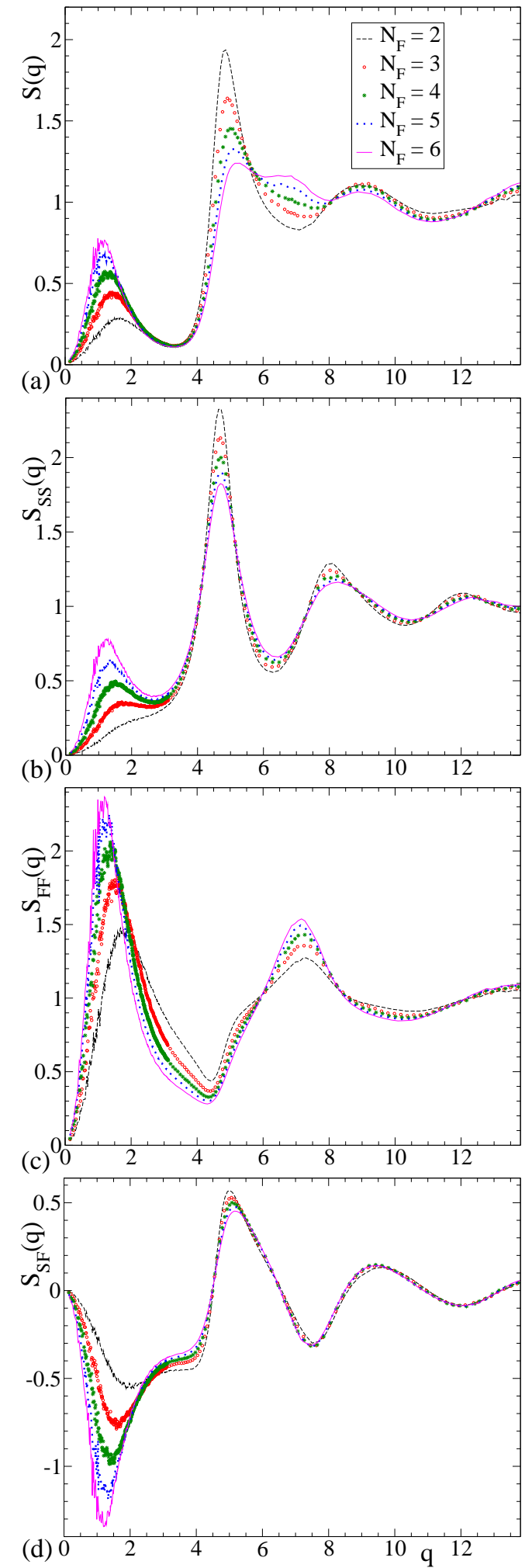

FIG. 4: For SF-combs at $T=0.30$, total and partial static structure factors for different arm length $N_{\mathrm{F}}$ [see legend in panel (a), same symbol codes for the other panels]. (a): total $S(q)$; (b): $S_{\mathrm{SS}}(q)$; (c): $S_{\mathrm{FF}}(q) ;(\mathrm{d}): S_{\mathrm{SF}}(q)$. 
enhancement of the intensity of this peak in the partially deuterated sample (note that Figure 2 is plotted in absolute units) is presumably due to the strong contribution corresponding to the cross-correlations between main chains and arms, that add positively due to the negative scattering length of the $\mathrm{H}$-atoms in the arms. This provides an additional evidence for the nanodomain structure in the real system. We suggest that the peak at $q_{\text {I }}$ will also remain under partial deuteration of the arms, in this case mainly reflecting the scattering arising from the correlations between domains of arm monomers and again the positively added cross-terms.

The second peak of $S_{\alpha \beta}(q)$ at $q_{\mathrm{II}} \sim 4.7,7.2$ and 5.1 for respectively S-S, F-F and S-F pairs, corresponds to nearest-neighbor distances between such pairs. Indeed the respective values of $2 \pi / q_{\mathrm{II}}$ correspond to $\approx 0.9 \sigma_{\alpha \beta}$, with $\sigma_{\alpha \beta}$ the diameters of the WCA interactions (eq 1). Moreover, the value of $q_{\mathrm{II}}$ is almost independent of the arm length, as expected for nearest-neighbor distances. The total structure factor $S(q)$ just includes all these nearestneighbor correlations, which lead to a double peak for the largest values of $N_{\mathrm{F}}$ (Figure $4 \mathrm{a}$ ). Finally, the observed oscillations at larger $q$-values have no major physical meaning and just correspond to higher-order harmonics.

The peak at $q_{\text {II }} \sim 1.3 \AA^{-1}$ in the experiments [21] (Figure 2) was enhanced by increasing the arm length, and was supressed under protonation of the arms. On this basis, the peak was assigned to correlations between arm monomers within the alkyl domains. The counterpart of the former effects in the simulated systems is the emergence, on increasing the arm length, of the peak at $q \sim 7.2$ in $S(q)$ (Figure $4 \mathrm{a}$ ), and its absence in $S_{\mathrm{SS}}(q)$ (Figure 4b). As noted above, $q \sim 7.2$ reflects correlations between nearest-neighbor FF pairs (Figure 4c). The corresponding peak at $q_{\mathrm{II}} \sim 4.7$ for S-S correlations does not have a clear direct counterpart in the experiments. In the simulated bead-spring model, $q_{\mathrm{II}}$ reflects nearest-neighbor distances of both intermolecular and intramolecular character. In real systems, the intramolecular structure of the main chain is reflected by $q$-values probing interatomic distances. A maximum in $S(q)$ located at about $3 \AA^{-1}$ usually appears in chemically simple polymers (see, e. g. [45]), that is attributed to typical intramolecular distances. Such $q$-values are much larger than the corresponding experimental peak for correlations between arm units at $q_{\mathrm{II}} \sim 1.3 \AA^{-1}$. This is not the case in the simulated comb copolymers due to their coarse-grained character.

Segregation in block copolymer systems is usually understood in terms of energetic un- 

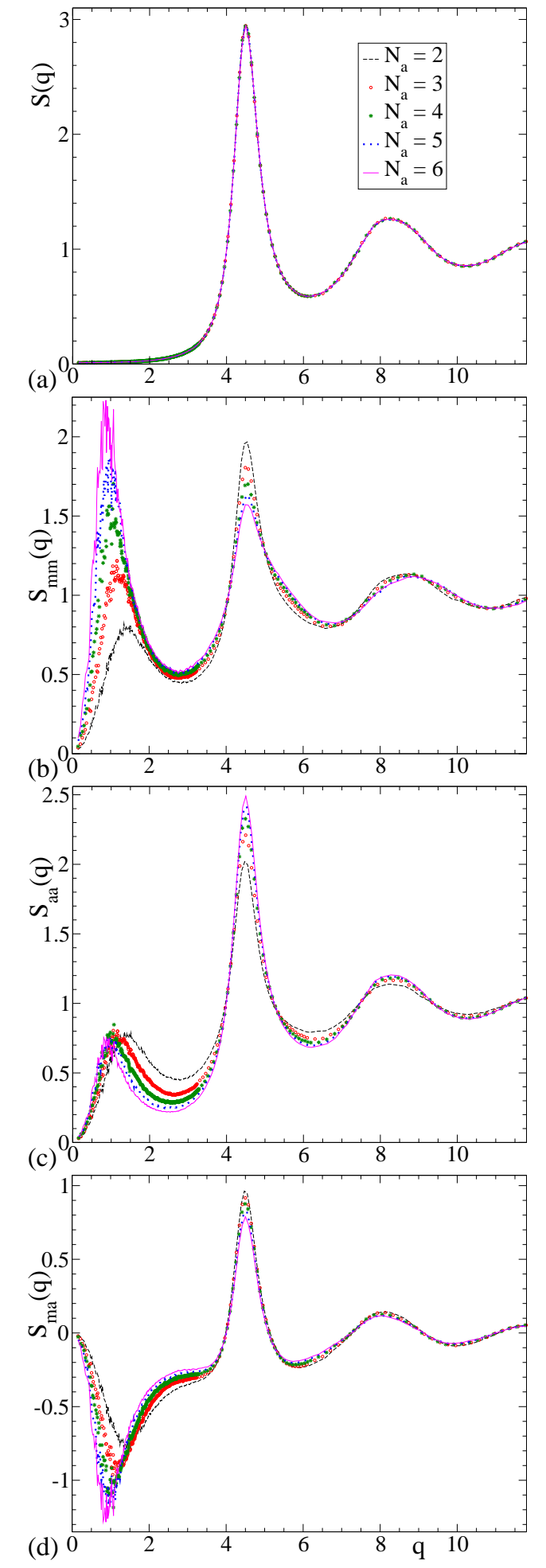

FIG. 5: For comb-like S-homopolymers at $T=0.60$, total and partial static structure factors for different arm length $N_{\text {a }}$ [see legend in panel (a), same symbol codes for the other panels]. Subindices $m$ and $a$ for the partial static structure factors denote monomers located, respectively, at the main chain and at the arms. (a): total $S(q) ;(\mathrm{b}): S_{m m}(q) ;(\mathrm{c}): S_{a a}(q) ;(\mathrm{d}): S_{m a}(q)$. 
compatibility between the two components. However, the former can also be driven by entropic effects $[46,47]$. This is the case for the investigated SF-combs, for which we take identical energy scales $\epsilon_{\alpha \beta}=\epsilon$, but distinct interaction diameters $\sigma_{\alpha \beta}$ (see above). Now we show that entropically driven segregation is possible even for one-component combs. Figure 5 shows static structure factors for pure S-combs of different arm length. We denote Smonomers in the main chain and in the arms as respectively $m$ and $a$. Panel (a) displays the total $S(q)$, i.e, including all correlations. Panels (b)-(d) show the partial contributions $S_{\alpha \beta}$ with $\alpha, \beta \in\{m, a\}$. The total $S(q)$ exhibits the same usual features of linear homopolymers. No structure is present below the nearest-neighbor peak at $q \sim 4.5$, and $S(q)$ decreases monotonically to $S(q \rightarrow 0) \sim 10^{-2}$, reflecting a very weak compressibility. As expected for a pure system, the position and intensity of the nearest-neighbor peak is esentially independent on the macromolecular weight. Having noted the standard behavior of $S(q)$, the partial contributions of $S_{\alpha \beta}$ instead reveal a striking feature. As for the case of the SF-combs, a prepeak arises and exhibits the same qualitative trends on increasing the arm length. Thus, it increases in intensity and shifts to lower $q$, from $q \approx 1.4$ for arms of $N_{\mathrm{a}}=2$ S-monomers to $q \approx 0.9$ for $N_{\mathrm{a}}=6$. As in Figure $4 \mathrm{~d}$ for SF-combs, $S_{m a}(q)$ exhibits an anticorrelation peak for $m-a$ pairs. This anticorrelation exactly cancels the positive correlations for $m-m$ and $a-a$ pairs, and the prepeak is absent in the total $S(q)$. This is not the case for the total $S(q)$ of the SF-combs (Figure 4a), where only partial cancelation occurs, due to a complex interplay of the different interaction diameters involved in the partial correlations.

The former results clearly reflect the organization of the pure combs in 'chemically identical' domains constituted by main-chain monomers or by arm monomers, in full analogy with the S- and F-domains observed for the SF-combs. This is confirmed by visual inspection of snaphots of the system (not shown), which exhibit morphologies analogous to that of Figure 3. As noted above, segregation in this case is of purely entropic nature, since all monomers in the system are identical. We suggest that it will generally arise in pure combs provided that the density of branch points is large enough. This would be confirmed by comparing neutron scattering structure factors in fully and partially deuterated samples. The prepeak, absent in the fully deuterated sample, would arise under partial deuteration of the main chain or of the arms. 


\section{DYNAMICS}

\section{A. General observations}

Neutron spin echo (NSE) experiments were carried out on the fully deuterated PnMA samples at the two maxima, $q_{\mathrm{I}}$ characteristic for inter-domain correlations and $q_{\mathrm{II}}$ revealing inter-monomer correlations within the alkyl nanodomains. To a good approximation [48], NSE measurements on fully deuterated samples access the normalized dynamic structure factor $F(q, t)=\left\langle\rho_{\mathbf{q}}(t) \rho_{-\mathbf{q}}(0)\right\rangle / S(q)$. Additional NSE measurements were carried out on the main-chain deuterated PHMA sample at $q=1.5 \AA^{-1} \approx q_{\mathrm{II}}$. In this case, the NSE signal is dominated [48] by the incoherent scattering function corresponding to the hydrogen atoms in the arms, $F_{\mathrm{H}_{\mathrm{a}}}^{\mathrm{s}}(q, t)=\left\langle\sum_{j=1}^{N_{\mathrm{H}_{\mathrm{a}}}} \exp \left[i \mathbf{q} \cdot\left(\mathbf{r}_{j}(t)-\mathbf{r}_{j}(0)\right)\right]\right\rangle / N_{\mathrm{H}_{\mathrm{a}}}$, where $N_{\mathrm{H}_{\mathrm{a}}}$ is the total number of protons in the arms.

By applying the rheological shift factors to the time scale [21], the NSE spectra at $q_{\mathrm{I}}$ collapse into a master curve that, for $t>5 \mathrm{ps}$, can be well described by a stretched exponential function

$$
F(q, t)=A_{q} \exp \left[-(t / \tau)^{\beta}\right]
$$

In this equation $A_{q}$ is the plateau height, $\tau$ is the relaxation time and $\beta<1$ is the stretching exponent. We find $\beta$-values close to 0.5 [21]. Such values are usually found for the structural relaxation in regular real polymers [48]. On the contrary, the correlators for collective motion at $q_{\mathrm{II}}$ and for self-motions of the arm units show extremely stretched functional forms, as can be seen in Figure 6. Free fits to eq 5 deliver unphysical values of $A_{q}>1$ and $\beta$-values of $\approx 0.1$. By fixing $A_{q}=1$, still very small $\beta$-values are obtained. Logarithmic-like decays provide a similar or even better description of the data [21, 22] (dashed line in Figure 6). Moreover, a marked decoupling between coherent and incoherent scattering is observed for the arm units: self-motions are much faster than collective dynamics, clearly beyond the standard de Gennes-like narrowing [42].

Finally, Figure 6 also shows the huge dynamic asymmetry present in the system. In the temperature range where the arm dynamics is accessible to the NSE window, the structural relaxation observed at the peak $q_{\mathrm{I}}=0.4 \AA^{-1}$ cannot be resolved. Based on the time/temperature superposition principle (that works at this peak [21]), and starting from the coherent data at $q_{\mathrm{I}}$ and $T=480 \mathrm{~K}$, we have estimated the curve corresponding to $q_{\mathrm{I}}$ and 


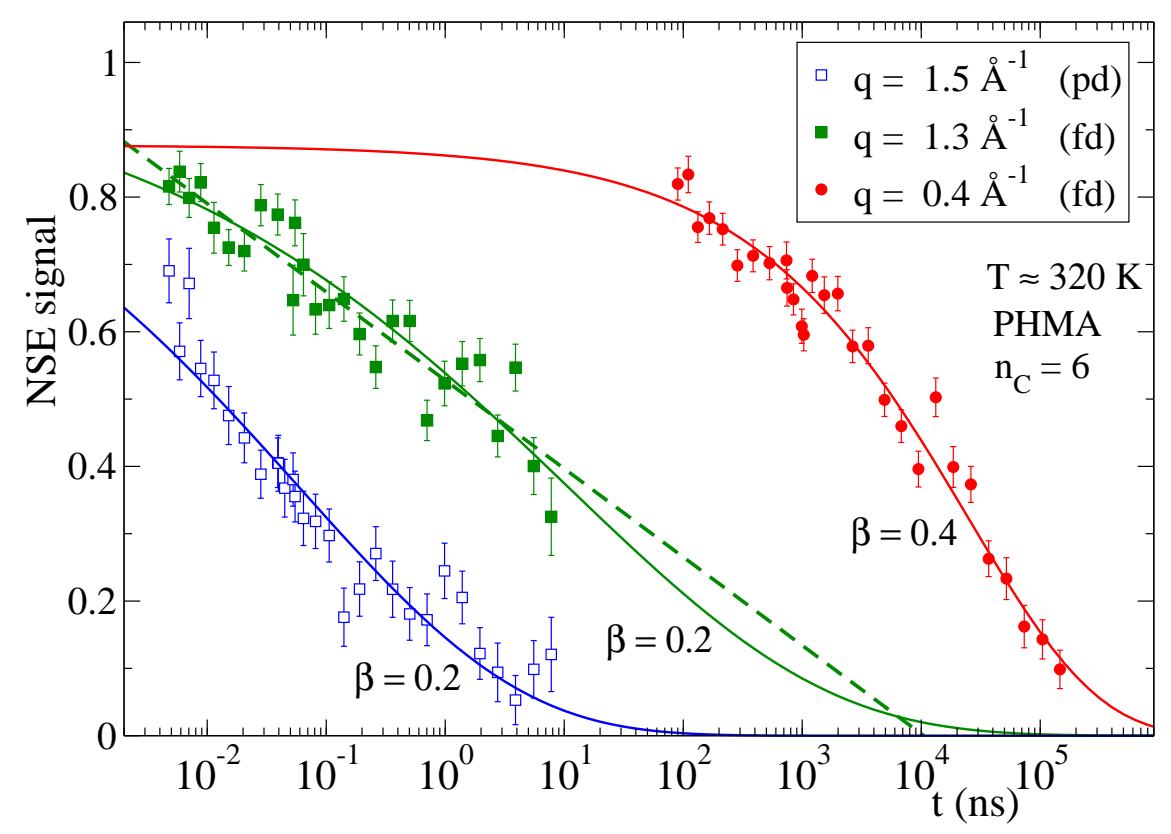

FIG. 6: NSE signal measured on fully deuterated (fd, full symbols) and partially deuterated (pd, empty squares) samples of PHMA. The data for the pd-sample corresponds to $q=1.5 \AA^{-1} \approx q_{\mathrm{II}}$ and $T=310 \mathrm{~K}$. The data for the fd-sample correspond to: i) $q_{\mathrm{II}}=1.3 \AA^{-1}$ and $T=330 \mathrm{~K}$ (full squares), ii) $q_{\mathrm{I}}=0.4 \AA^{-1}$ and $T=480 \mathrm{~K}$ (full circles). The time scale of ii) has been shifted, by applying time/temperature superposition (see [21]), in order to estimate the corresponding data at $T=320 \mathrm{~K}$. This allows for a direct comparison with the other data sets in the figure. Solid lines are fits to stretched exponentials (eq 5). The obtained $\beta$-values are indicated. The dashed line is a logarithmic description [21, 22].

$T=320 \mathrm{~K}$. The difference in the characteristic times for the decays of the coherent functions at $q_{\mathrm{I}}$ and $q_{\mathrm{II}}$ is of about 3 decades. Thus, a very large dynamic asymmetry is present in this system. This asymmetry becomes more pronounced with decreasing temperature [21, 22].

Figure 7 shows simulated counterparts of the coherent functions in SF-combs, for fixed arm length $N_{\mathrm{F}}=6$. We present both the total coherent function $F(q, t)=$ $\left\langle\rho_{\mathbf{q}}(t) \rho_{-\mathbf{q}}(0)\right\rangle / S(q)$ and the partial contributions $F_{\alpha \alpha}(q, t)=\left\langle\rho_{\mathbf{q}}^{\alpha}(t) \rho_{-\mathbf{q}}^{\alpha}(0)\right\rangle / S_{\alpha \alpha}(q)$ (with $\alpha \in\{\mathrm{S}, \mathrm{F}\}$ ). The panel (a) shows data for $q=1.30$, which approximately corresponds to the peak at $q_{\mathrm{I}}$ in the static structure factors. The panel (b) shows data for $q=7.00$, which approximately corresponds to the peak at $q_{\mathrm{II}}$ for nearest-neighbor F-F correlations (Figure 4). The results for $q \approx q_{\text {I }}$ show almost no differences between the (normalized) total and partial 

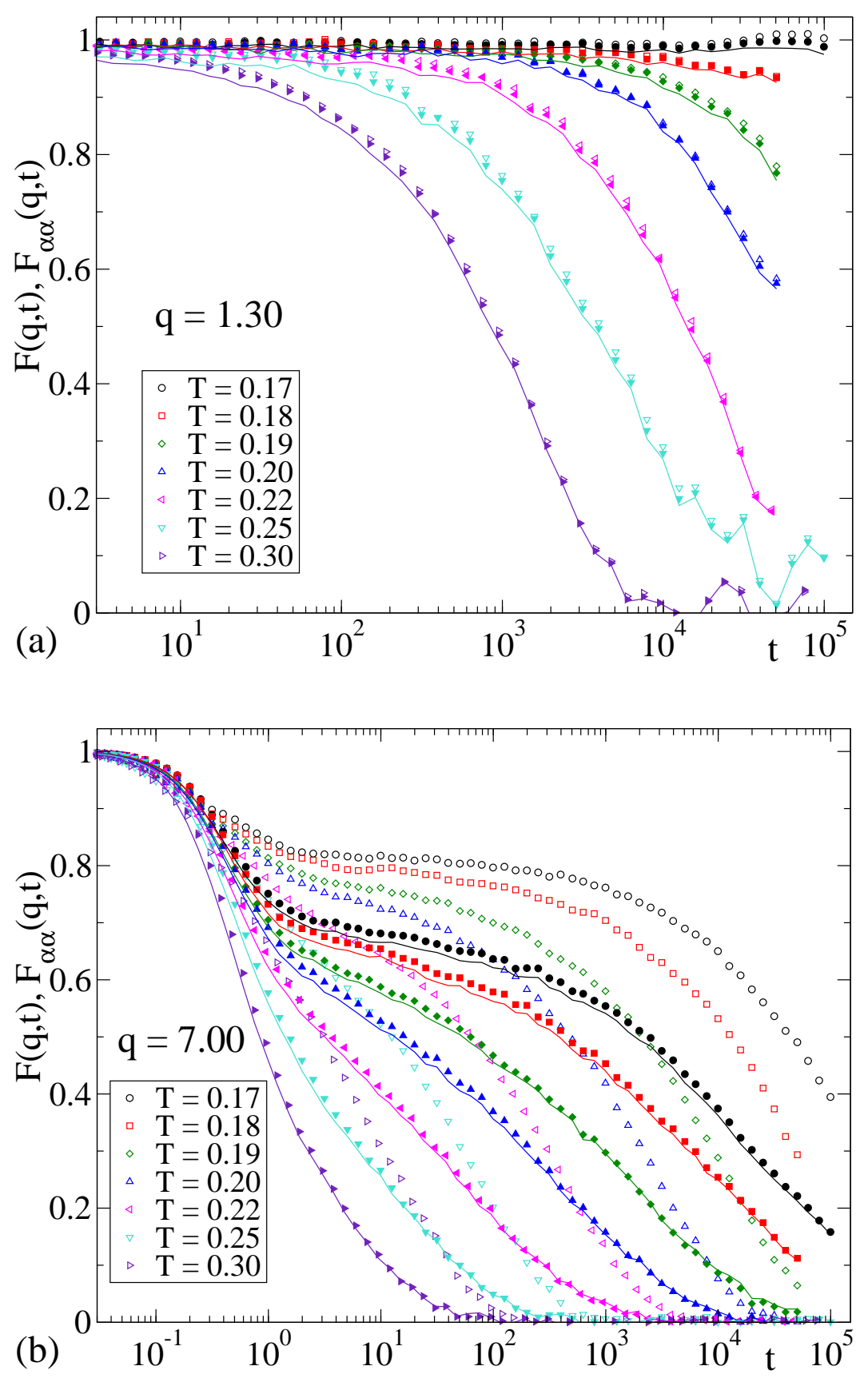

FIG. 7: Coherent scattering functions for SF-combs of arm length $N_{\mathrm{F}}=6$ at wave vectors $q=1.30$ (a) and $q=7.00$ (b). Different colors correspond to different temperatures (see legend). Empty and full symbols correspond respectively to S-S and F-F correlations. Lines correspond to all correlations. 
coherent scattering functions. This confirms that the experimental coherent function at $q_{\mathrm{I}}$ is a good approximation for the main-chain/main-chain dynamic structure factor, as pointed out in $[21,22]$. Having noted this and as discussed above for the statics, the peak at $q_{\text {I }}$ does not only reflect collective correlations between main-chain units, but also between domains of arm monomers. In fact, the respective coherent functions are almost identical at $q_{\mathrm{I}}$.

In the case of the second peak at $q_{\mathrm{II}} \approx 7.0$ we find $F(q, t) \approx F_{\mathrm{FF}}(q, t)$, i.e., the total coherent function at $q_{\mathrm{II}}$ essentially reflects collective correlations between arm monomers, confirming the experimental interpretation [21]. The coherent functions $F_{\mathrm{SS}}(q, t)$ at $q_{\mathrm{II}} \approx 7.0$ exhibit slower relaxation than the respective functions $F_{\mathrm{FF}}(q, t)$ at the same temperature (Figure 7b). Moreover the time scale separation between both functions increases on decreasing temperature. This reflects an increasing dynamic asymmetry in the SF-combs, with the arm F-monomers showing faster dynamics. These features are also found in the real systems (see above).

The decay from the plateau can be well described by a stretched exponential function (eq 5). Figure 8 a shows some representative fits for $F(q, t)$ at $q_{\mathrm{I}} \approx 1.3$ and $q_{\mathrm{II}} \approx 7.0$. In agreement with the experimental findings for the total coherent function, relaxation is much more stretched at $q_{\mathrm{II}}(\beta \approx 0.4)$ than at $q_{\mathrm{I}}(\beta \approx 0.7)$. These $\beta$-values are larger than those found in the experiments (see Figure 6). This difference with real polymers is a general finding, even for linear homopolymers. Thus, at the $q$ for the maximum of $S(q)$ one finds values $\beta \approx 0.7$ for fully-flexible bead-spring chains [35-37], while $\beta \approx 0.5$ is typically found in real homopolymers [48]. The difference is in part due to the absence of intramolecular barriers in the simulated model. Indeed lower $\beta$-values are found when barriers are implemented in the model [49]. Having noted this the former results show that, as found in the experiments (see above), the structural relaxation monitored at the peak $q_{\mathrm{I}}$ exhibits 'standard' features, in the meaning that stretching (here $\beta \approx 0.7$ ) is similar to that found in homopolymers. This behavior applies for the collective relaxation of both the main-chain and arm correlations at this inter-domain level. We also note that the decay of $F_{\mathrm{SS}}\left(q_{\mathrm{II}}, t\right)$ is also 'standard' (again $\beta \approx 0.7$ ), an information that cannot be accessed experimentally.

The experimental results at $q_{\mathrm{II}}$ seem compatible with a quasi-logarithmic functional form for arm relaxation $[21,22]$. In view of the simulations, this conclusion must be taken with care due to the limited statistics and time window of the data in Refs. [21, 22]. Panel (b) of 

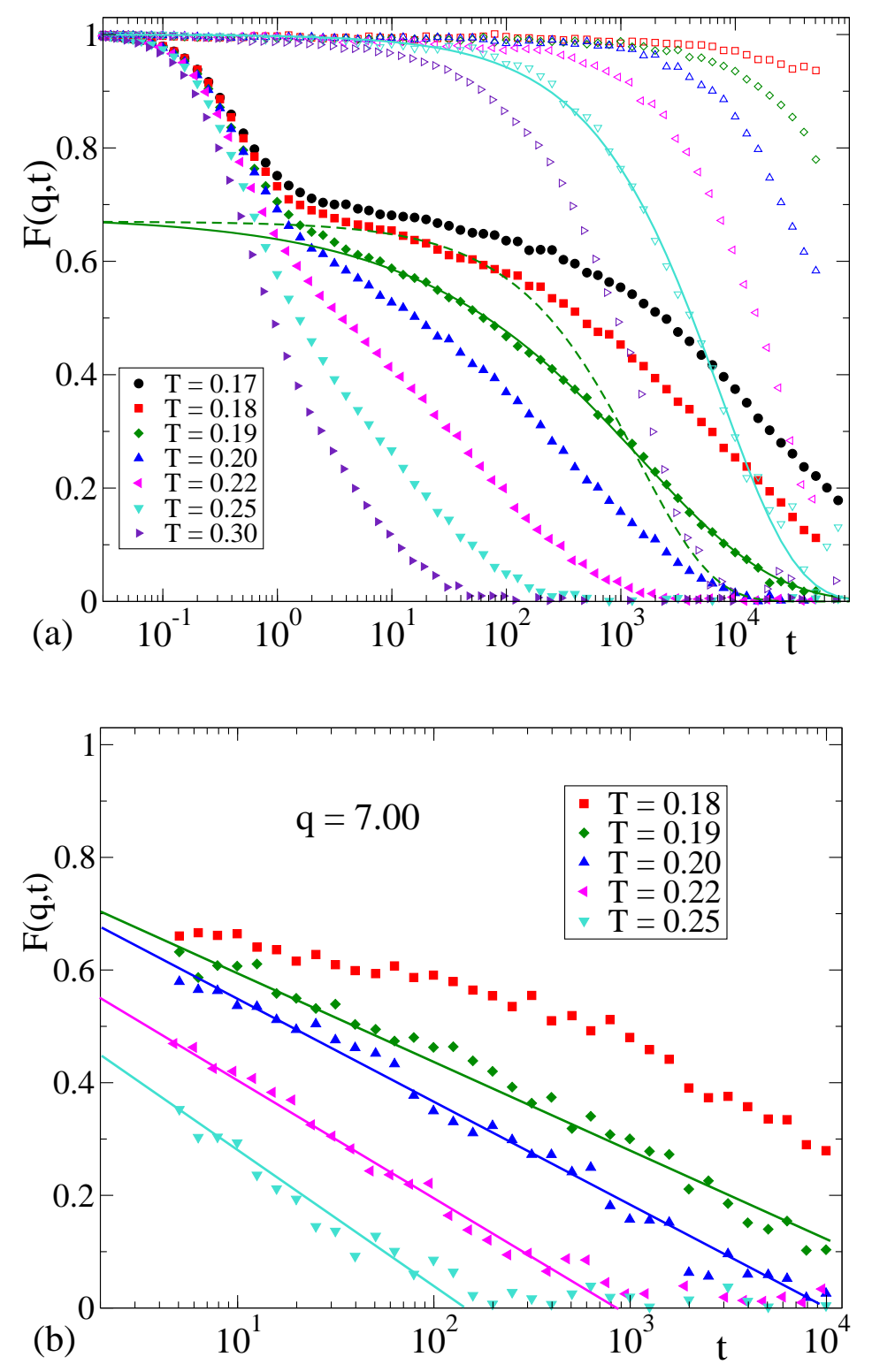

FIG. 8: Coherent scattering functions $F(q, t)$ for SF-combs of arm length $N_{\mathrm{F}}=6$. Full symbols: $q=7.00$. Empty symbols: $q=1.30$. Same symbol codes correspond to same temperatures (see legend). Panel (a) shows data in the time window covered by the simulations, and averaged over 20 time origins. Panel (b) shows data for $q=7.00$, averaged over a single time origin and in a time window similar to that of experiments in Refs. [21, 22]. Straight lines in (b) correspond to hypothetical logarithmic decays. Solid lines in (a) are fits to stretched exponentials, with $\beta=0.66$ and 0.38 for respectively $q=1.30$ and $q=7.00$. The dashed line is a stretched exponential with the same relaxation time $\tau$ of the former free fit for $q=7.00$. The exponent for the dashed line is $\beta=0.68$, the same observed for the F-homopolymer at this $q$ and at temperatures with similar $\tau$. 
Figure 8 displays the same data sets of $F(q, t)$ in panel (a), but in a representation aimed to mimic the conditions of the experimental data. Whereas data in panel (a) are averaged over 20 time origins (see Section II), data in panel (b) correspond to a single time origin, with a statistical noise similar to the experimental data. In bead-spring models the time unit (here $\tau_{\mathrm{sim}}=\sigma_{\mathrm{FF}}(m / \epsilon)^{1 / 2}$ ) can be qualitatively mapped to $\tau_{\exp } \sim 1$ ps [36]. Note that both time scales are roughly the respective ones in bead-spring and real polymer melts for the onset of the plateau regime $[36,48]$. With the former transformation the results of panel (b) are represented for an approximate time window of $5 \mathrm{ps}<t<10 \mathrm{~ns}$, which corresponds to the experimental data of Refs. [21, 22]. With the representation of Figure 8b, the data are compatible with logarithmic decays, as proposed in [21, 22]. However, this feature is discarded when data are represented with good statistics and over a broader time window (Figure 8a). Having noted this, we confirm the experimental observation [21, 22] of a much stronger stretching $(\beta \approx 0.4)$ at $q=q_{\mathrm{II}}$ (where $\left.F(q, t) \approx F_{\mathrm{FF}}(q, t)\right)$ than for the linear F-homopolymer $(\beta \approx 0.7)$.

\section{B. Influence of the arm length}

The influence of the arm length on the dynamics of each subsystem can be easily monitored by calculating mean squared displacements (MSD, $\left\langle\Delta r^{2}(t)\right\rangle$ ), which are not experimentally accessible. Figure 9 shows results for the monomer MSD in SF-combs, at fixed temperature, as a function of the arm length. Data for the S15 and F15 homopolymers are included for comparison. Mixing with the other species have opposite effects for the S- and F-monomers. In the system with the shortest arms, $N_{\mathrm{F}}=2$, the fraction of F-monomers is close to the $50 \%$ (see Table I). In analogy with observations in polymer blends [50, 51], this degree of mixing dramatically accelerates the dynamics of the S-monomers. This effect persists on further increasing the arm length. The observed plasticization effect is in agreement with the experimental finding for main chain dynamics in PnMAs on increasing the length of the alkyl groups [22]. In the case of the F-monomers, the dynamics are instead strongly slowed down respect to the F-homopolymer by increasing the concentration of the slow Smonomers, i.e., by decreasing the arm length (see panel (b)). This is again in agreement with the experimental observation [22]. 

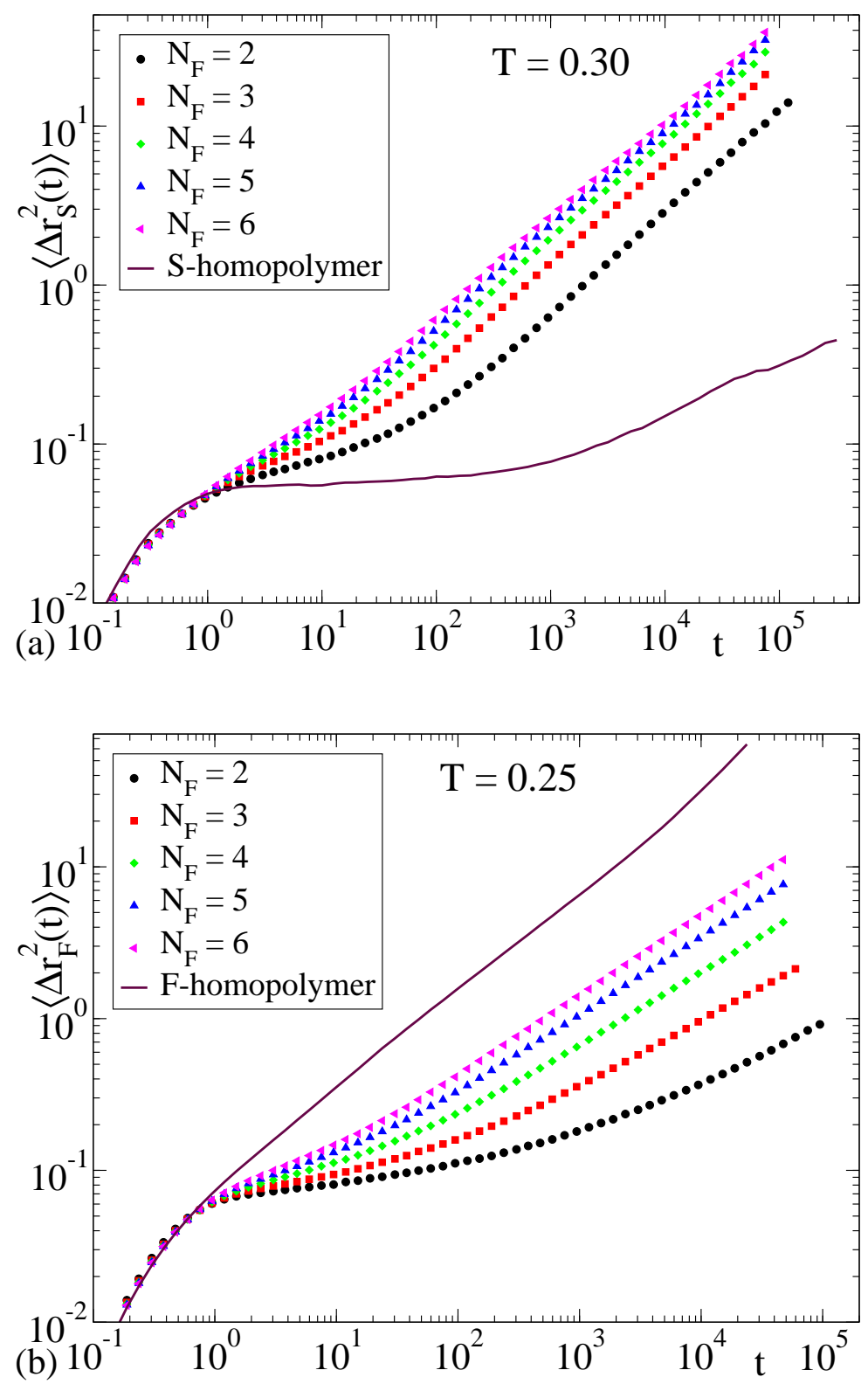

FIG. 9: (a): Symbols: mean squared displacement for the S-monomers in SF-combs of several arm lengths (see legend) at fixed $T=0.30$. The line represents the corresponding data for the S-homopolymer. (b): as panel (a) for the F-monomers, at fixed $T=0.25$

\section{Temperature dependence}

Figure 10 shows the temperature dependence of the MSD in SF-combs of fixed arm length $N_{\mathrm{F}}=6$. As usual in glass-forming systems, on decreasing temperature the caging regime (i.e., the plateau arising at $t \sim 1$ ) extends over longer time scales. At fixed $T$, caging is 


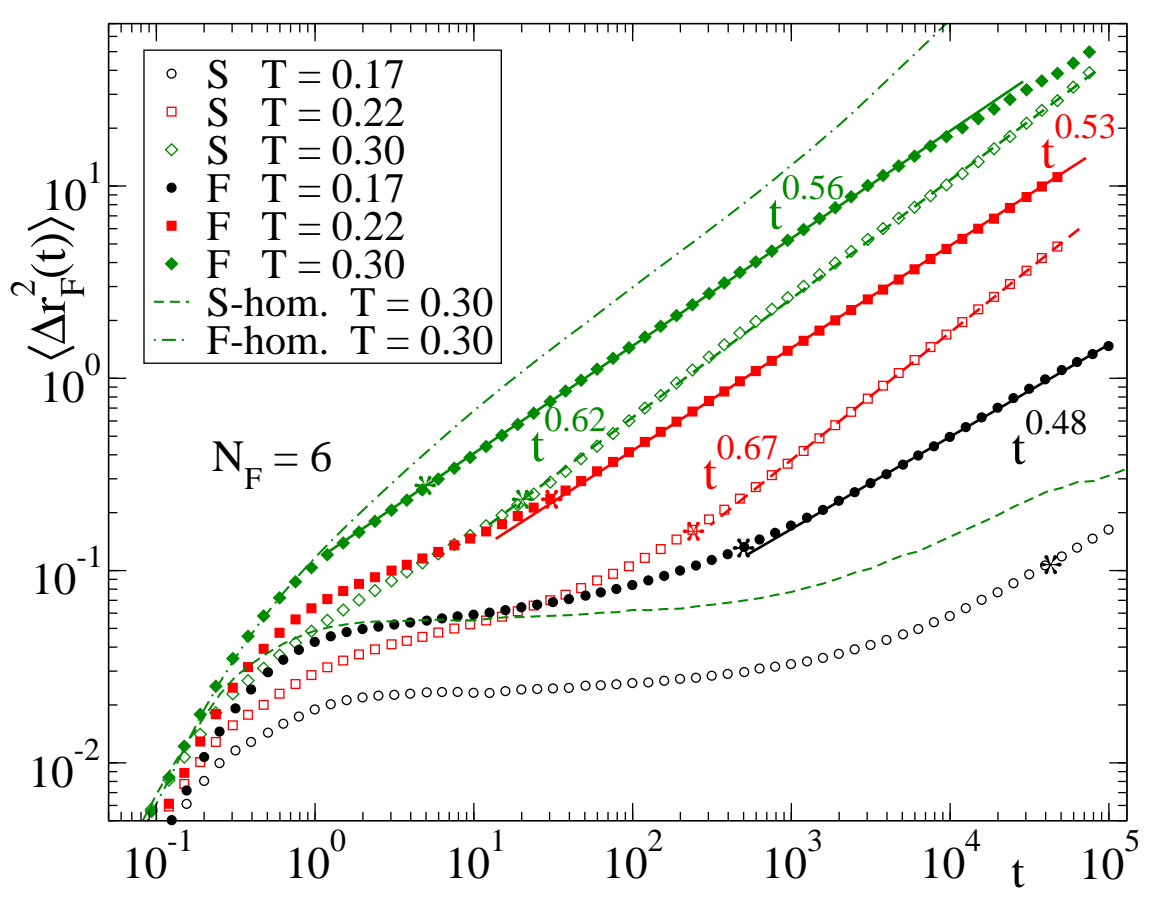

FIG. 10: Symbols: MSD of S- and F-monomers in SF-combs of arm length $N_{\mathrm{F}}=6$. Data are shown for different temperatures (see legend). For comparison, we include the MSD, at $T=0.30$, of the homopolymers S15 (dashed line) and F15 (dashed-dotted line). Straight lines represent approximate power laws $\left\langle\Delta r_{\beta}^{2}(t)\right\rangle \propto t^{x}$ (exponents are indicated for each data set). Stars indicate the times for the maxima of the respective non-Gaussian parameters (see below).

more pronounced for the slow S-component. For comparison, we include the MSD of the homopolymers S15 and F15 at $T=0.30$. As anticipated in Section II, at a same $T$ the two homopolymers exhibit very different dynamics. We can estimate a characteristic time scale $\tau$ of the $\alpha$-relaxation as $\left\langle\Delta r_{\beta}^{2}(\tau)\right\rangle \sim \sigma_{\beta \beta}^{2}$ (with $\beta \in\{\mathrm{S}, \mathrm{F}\}$ ). Thus, the $\alpha$-time scales of both homopolymers at $T=0.30$ differ by more than four decades. Though much less pronounced, this dynamic separation is also present in the SF-combs. In analogy with observations in the scattering functions (Figure 7b) and in agreement with the experimental results on PnMAs [21, 22], dynamic asymmetry in the combs is enhanced by decreasing temperature. This effect is analogous to the general observation in polymer blends. However, unlike the case of polymer blends [50], the two components in the combs are linked by permanent chemical bonds. Due to this constraint, the MSD of the S- and F-monomers merge again at long times. From data in Figure 4 we can estimate the domain size for $N_{\mathrm{F}}=6$ as 


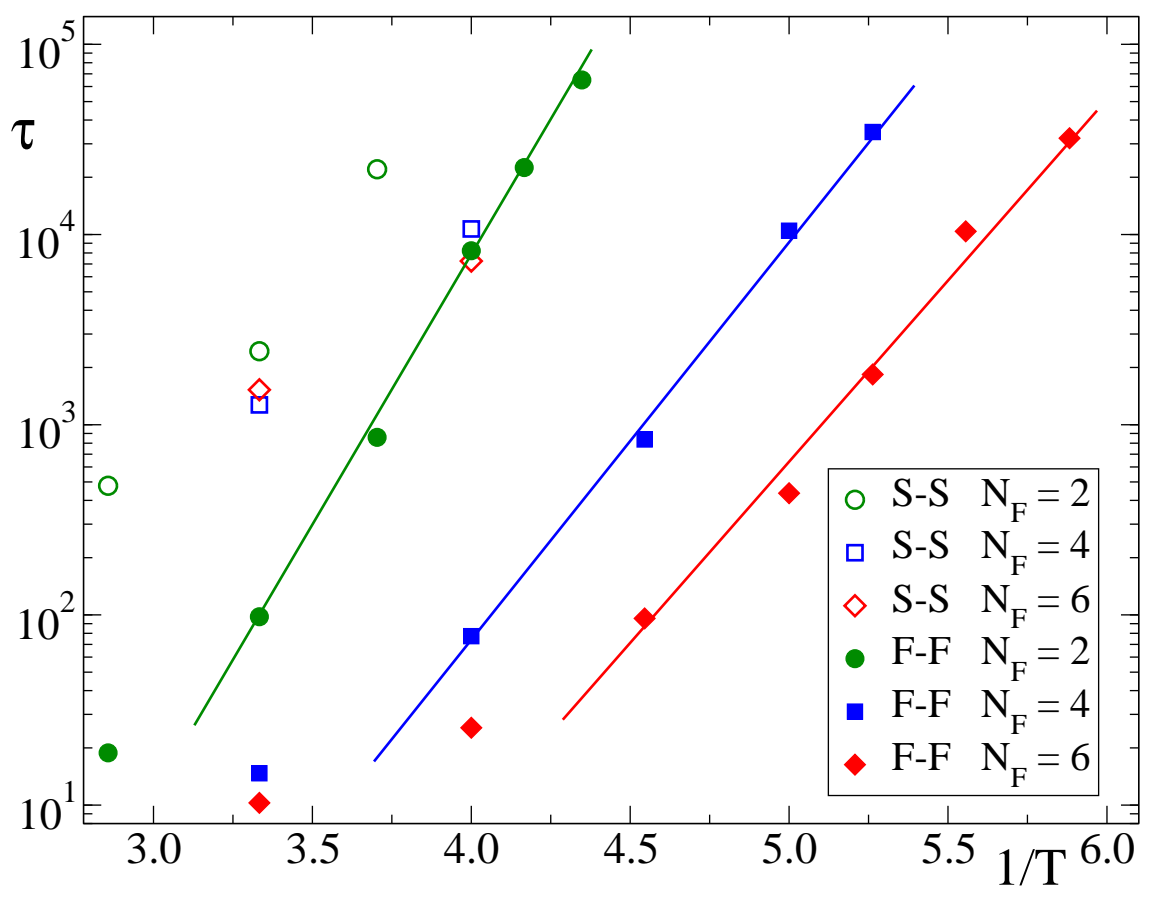

FIG. 11: Temperature dependence of the relaxation times (see text) of coherent scattering functions. Empty symbols correspond to S-S correlations at $q=1.30$. Full symbols correspond to F-F correlations at $q=7.00$. Data are shown for arm lengths $N_{\mathrm{F}}=2,4$, and 6 (see legend). Lines show effective Arrhenius descriptions.

$d \approx 2 \pi / q_{\mathrm{I}} \approx 6$. We observe that the merging of the MSD of both species is almost complete at $\left\langle\Delta r_{\beta}^{2}\right\rangle \sim d^{2}$, i.e, at time scales probing the domain size. An analogous observation has been recently reported from simulations of poly(vinyl pyrrolidone), a homopolymer with a relatively simple but bulky side group [52]. Presumably, full merging of the MSD of S- and Fmonomers will take place at the onset of the diffusive regime $\left\langle\Delta r_{\beta}^{2}(t)\right\rangle \propto t$, which is beyond the simulation time window. Prior to that, the MSD of both species shows subdiffusive behavior, $\left\langle\Delta r_{\beta}^{2}(t)\right\rangle \propto t^{x}$ with $x<1$. The effective exponents $x$ seem to follow different trends on decreasing temperature. Thus, $x$ increases and decreases for respectively the Sand F-component.

Subdiffusive power laws in the MSD are present in many physical situations. A wellknown example is given by chain dynamics in polymer systems. For the simple case of linear chains, the decaging process is followed by Rouse dynamics [35-37]. Further sublinear regimes arise after the Rouse regime in the case of strongly entangled chains [53]. In the 
limit of long chains, the Rouse model predicts an effective exponent $x=0.5$. Due to finite size effects [53], linear bead-spring chains of $N \sim 15$ monomers show values $x \gtrsim 0.6$. Results in Figure 10 for the S-monomers in the main chain show such values and seem compatible with motions dominated by Rouse dynamics.

For the F-monomers, a rationalization of the subdiffusive regime in terms of Rouse dynamics does not seem plausible. Indeed we observe analogous features and similar exponents $x \sim 0.5$ even for arms of $N_{\mathrm{F}}=2$. Clearly, this small length scale cannot be captured by the Rouse model, which explicitly neglects excluded volume effects [53]. A different scenario for the motion of the F-monomers may be invoked in relation with the problem of dynamics in crowded environments. The motion along networks of interconnected channels in slow matrixes usually displays subdiffusive behavior $\left\langle\Delta r_{\beta}^{2}\right\rangle \propto t^{x}$, with $x<1$, over several time decades [54-58]. The morphology of the nanodomains (Figure 3) and the slow nature of the domains rich in S-monomers suggests a related scenario for the motion of the F-monomers.

The MSD is not accessible by neutron scattering experiments. However, the former observations seem compatible with the strong stretching exhibited by the incoherent function of the H-atoms in the alkyl group (see Figure 6). The MSD can be estimated by invoking the Gaussian approximation [48], $\left\langle\Delta r_{\mathrm{H}_{\mathrm{a}}}^{2}(t)\right\rangle=-\left(6 / q^{2}\right) \ln \left[F_{\mathrm{H}_{\mathrm{a}}}^{\mathrm{s}}(q, t)\right]$. Deviations from Gaussian behavior may be expected (they indeed become clear in the simulations, see below). Still, by applying the Gaussian approximation as a crude estimation, strong stretching in $F_{\mathrm{H}_{\mathrm{a}}}^{\mathrm{s}}(q, t)$ points to small $x$-values in the corresponding MSD.

Figure 11 shows for the two species in the simulated SF-combs, and for several arm lengths, the temperature dependence of the relaxation times $\tau$ of the coherent scattering functions at $q=1.30$ and $q=7.00$. For comparison with the experimental trends in $\operatorname{poly}(n$ alkyl methacrylates), we follow the same analysis as in Ref. [22]. Thus, we define $\tau$ as the time for which the normalized scattering function decays to $F_{\alpha \alpha}(q, \tau)=A_{q} e^{-1}$, with $A_{q}$ the plateau height. In Ref. [22] the latter was defined as the value of the function at a time $t_{0}$ around the beginning of the plateau. Following this criterium, we use $t_{0}=2$. As in Ref. [22], we represent $\tau$ in logarithmic scale versus $1 / T$. Data for the F-monomers are compatible with Arrhenius behavior $\tau \propto \exp \left(E / k_{\mathrm{B}} T\right)$ over 3 decades in time, though we cannot assess the validity of this description at lower temperatures. The apparent activation energy $E$ decreases on increasing the arm length, i.e., on increasing the fraction $x_{\mathrm{F}}$ and size of the F-domains. These trends are again consistent with the NSE experimental results in PnMAs, 

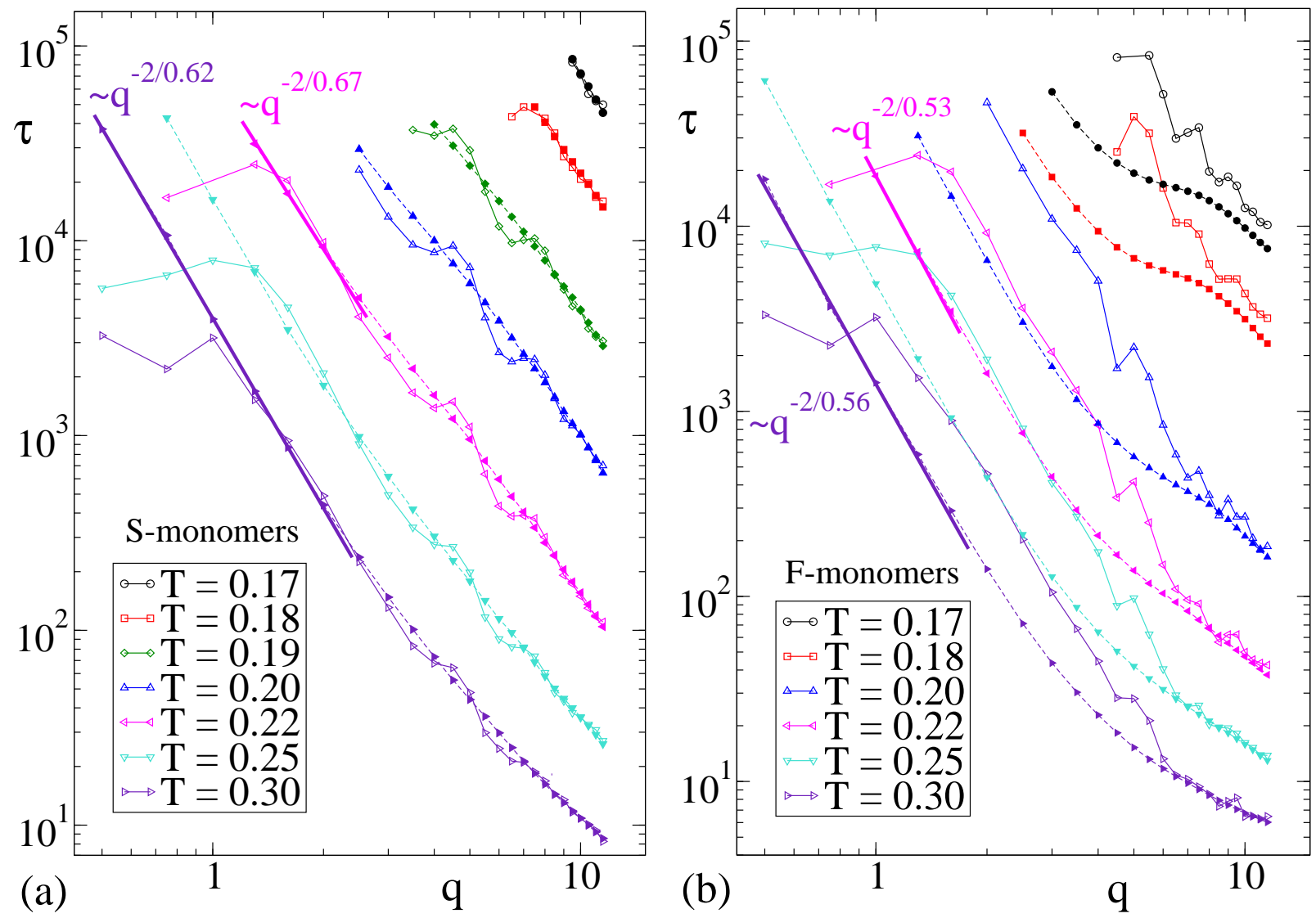

FIG. 12: For SF-combs with arm length $N_{\mathrm{F}}=6$, and at several temperatures, $q$-dependence of the relaxation times (see text) of scattering functions. (a): coherent S-S (empty symbols) and incoherent S (full symbols); (b): coherent F-F (empty symbols) and incoherent F (full symbols). Data sets with same codes for empty and full symbols correspond to a same temperature (see legend). Thin solid and dashed lines are guides for eyes. Thick solid lines correspond to approximate scaling $\tau \sim q^{-2 / x}$ (exponents are indicated).

addressing the high temperature range [22].

\section{Decoupling of self- and collective dynamics}

We have shown that the decoupling between self- and collective dynamics is one of the peculiarities exhibited by the arm units in PHMA (see Figure 6). Moreover, this effect is enhanced with decreasing temperature [22]. Now we show that this feature is also reproduced by the simulations. Figure 12 shows, for SF-combs of fixed $N_{\mathrm{F}}=6$ at several temperatures, 
the $q$-dependence of the relaxation times $\tau$ of scattering functions, according to the former definition of $\tau$ (see previous subsection). Data are presented for the incoherent scattering functions of both species, $F_{\mathrm{S}}^{\mathrm{s}}(q, t)$ and $F_{\mathrm{F}}^{\mathrm{s}}(q, t)$, and for the respective coherent functions for S-S and F-F correlations. The incoherent function is defined as $F_{\alpha}^{\mathrm{s}}(q, t)=\left\langle\sum_{j=1}^{N_{\text {mon }}^{\alpha}} \exp [i \mathbf{q}\right.$. $\left.\left(\mathbf{r}_{j}(t)-\mathbf{r}_{j}(0)\right]\right\rangle / N_{\text {mon }}^{\alpha}$. For $q<q_{\text {I }}$ we observe a progressive deviation of the coherent times below the incoherent ones. This is a general feature also present in linear homopolymers for length scales far beyond nearest-neighbor distances. Its origin is not well understood [65] and is beyond the scope of this work. For $q>q_{\mathrm{II}}$ there is almost no separation between coherent and incoherent times. This is not surprising, since $q_{\text {II }}$ reflects nearest-neighbor distances, and therefore no relevant collective correlations are probed for $q>q_{\mathrm{II}}$. For the range $q_{\mathrm{I}}<q<q_{\mathrm{II}}$, which probes the domain structure, the comparison between self- and collective dynamics reveals very different features for S- and F-monomers. Coherent and incoherent times for the S-monomers are very similar. By invoking theory of simple liquids, this may be understood esentially in terms of de Gennes narrowing [42]. On the contrary, a strong decoupling of self- and collective dynamics is observed for the F-monomers. In agreement with experiments [22], the decoupling is enhanced on decreasing $T$. At the lowest investigated temperatures coherent times are shifted by factors of up to 10 respect to the incoherent ones.

This feature for the F-monomers can be understood as a consequence of the slow rearrangement of the network of domains. This induces slow collective correlations between the F-monomers. However, the F-monomers explore their domains by fast self-motions, leading to the observed differences between coherent and incoherent times. As temperature decreases, rearrangement of the network becomes slower, and decoupling between self- and collective dynamics of F-monomers is enhanced. This effect for the fast component is present in other mixtures with strong dynamic asymmetry. Some examples are colloidal mixtures of large and small particles [59,60], polymer blends [50,61], mixtures of big and small stars [62], and alkali silicates [63, 64]. However, in these systems the observed decoupling is much stronger, of even 3-4 decades for similar simulation windows. The reason is that, unlike in the comb copolymers, dynamic asymmetry can be much stronger due to the absence of permanent links between the two components. The fast component can even show a finite diffusivity while the slow one is in the glassy state [62, 63]. This is clearly impossible for block copolymers (see mean squared displacements in Figure 10). 


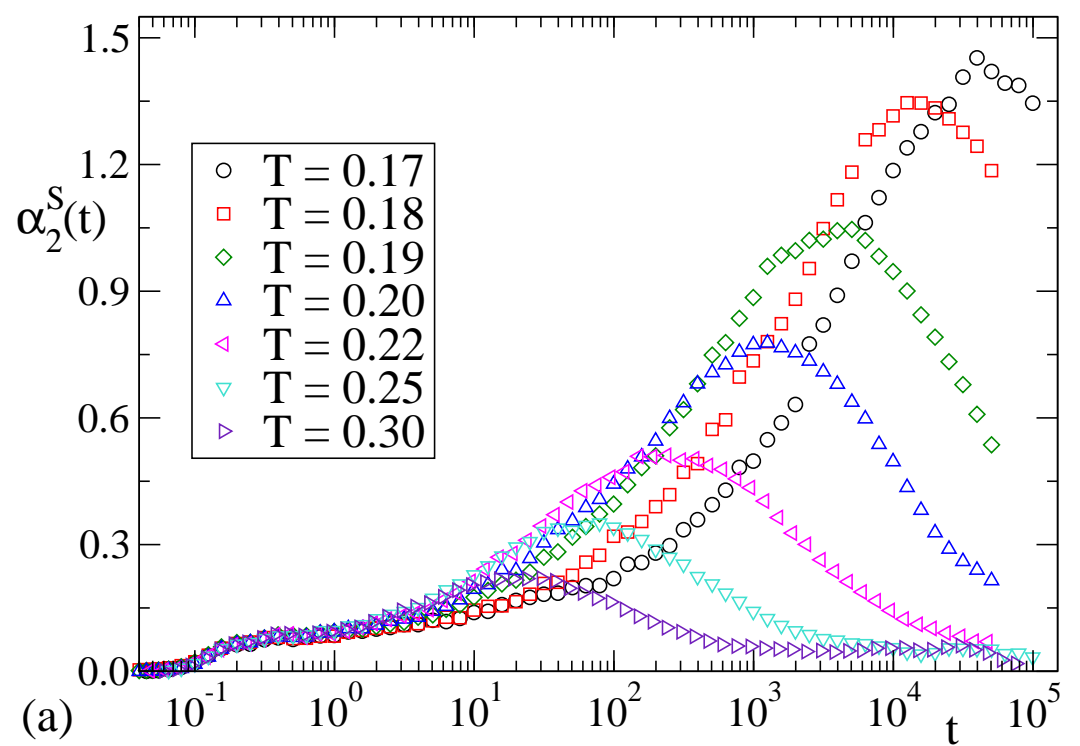

(a)

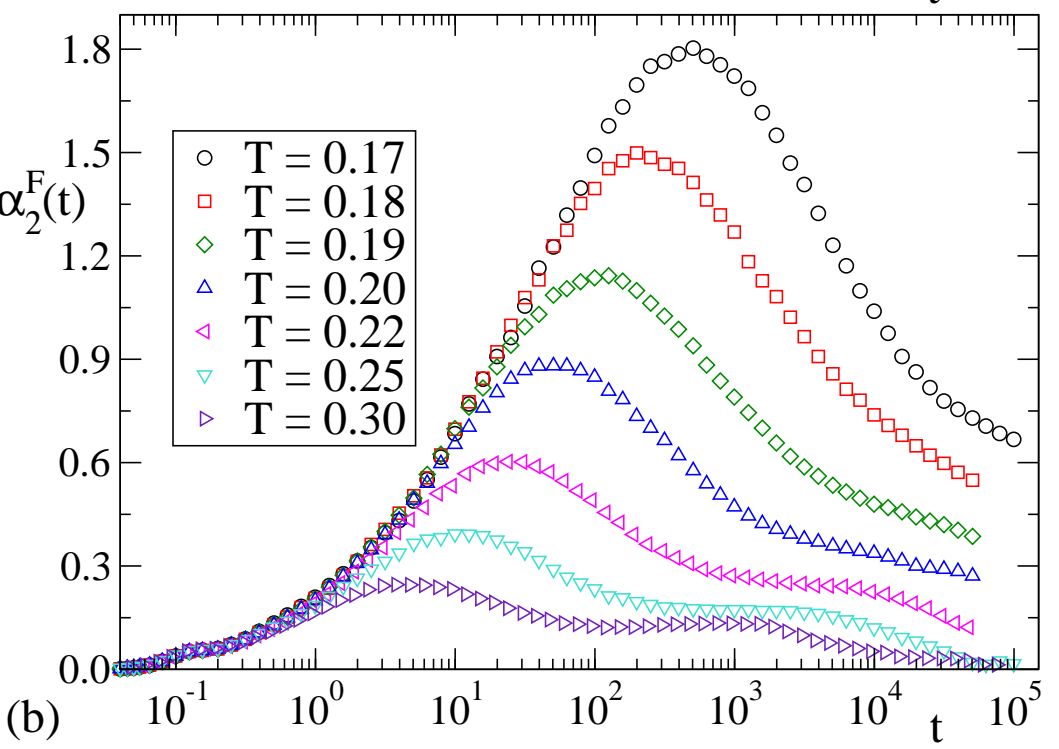

FIG. 13: Temperature dependence of the non-Gaussian parameter for SF-combs of $N_{\mathrm{F}}=6$. Panel (a): data for S-monomers. Panel (b): data for F-monomers.

\section{E. Dynamic heterogeneity}

In the limit of Gaussian dynamics the incoherent scattering function of the $\alpha$-species obeys $F_{\alpha}^{\mathrm{s}}(q, t)=\exp \left[-q^{2}\left\langle\Delta r_{\alpha}^{2}(t)\right\rangle / 6\right]$. According to this, if the MSD follows subdiffusive behavior $\left\langle\Delta r_{\alpha}^{2}(t)\right\rangle \sim t^{x}$, the relaxation time $\tau$ defined above will depend on $q$ as $\tau \sim q^{-2 / x}$. Figure 12 shows a test of this relation in SF-combs of $N_{\mathrm{F}}=6$, by taking the effective exponents $x$ of the mean squared displacements of Figure 10. For the S-monomers approximate Gaussian 
behavior is reached for wave vectors smaller than $q \sim 2.5$. The associated length scale $2 \pi / q$ is about $1.5 \sigma_{\mathrm{SS}}$. In other words, approximate Gaussian behavior is reached when the Sparticles move over a distance similar to their own size. This is indeed the usual observation in glass-forming systems [66]. Following these arguments, the crossover to Gaussian behavior of the F-monomers might be expected to occur at $q \sim 2 \pi /\left(1.5 \sigma_{\mathrm{FF}}\right) \approx 4$. However, it actually occurs at larger length scales, namely close to the peak at $q_{\mathrm{I}} \approx 1.3$ in the static structure factor (Figure 4). Thus, the Gaussian limit for the F-monomers is only approached by exploring the size of the nanodomains.

Deviations of the distribution of displacements from the Gaussian approximation can be quantified by means of the non-Gaussian parameter, $\alpha_{2}^{\beta}(t)=3\left\langle\Delta r_{\beta}^{4}(t)\right\rangle / 5\left\langle\Delta r_{\beta}^{2}(t)\right\rangle^{2}-1$, which is exactly zero for Gaussian dynamics. Figure 13 shows results for the non-Gaussian parameter of S- and F-monomers in SF-combs of arm length $N_{\mathrm{F}}=6$. A detailed comparison with the data of Figure 12 shows that the approximate power-law behavior $\tau \sim q^{-2 / x}$ is reached at time scales for which $\alpha_{2}^{\beta}$ decays to small values $\sim 0.1$. This is consistent with the interpretation of the former power law in terms of Gaussian behavior (see above). As usually observed in glass-formers [66], $\alpha_{2}^{\beta}$ increases from zero at $t=0$ up to a maximum. This maximum is located around the decaging time, i.e., at the crossover between the late caging regime and the early structural relaxation (see MSD in Figure 10). It reflects significant dynamic heterogeneity at that time scale $[66,67]$. As usual, this effect is strongly enhanced on decreasing temperature. At longer times dynamic heterogeneity is weaker and $\alpha_{2}^{\beta}$ decays. The Gaussian limit $\left(\alpha_{2}^{\beta}=0\right)$ is only reached within the simulation time window at the highest investigated temperatures. Prior to the final decay to zero, a plateau regime arises for the F-monomers. This effect is related to dynamic heterogeneity associated to the exploration of the F-domains, as discussed above.

Now we characterize dynamic heterogeneity of the F-component in more detail by computing dynamic correlators for specific monomers and bonds. Panels (a) and (b) of Figure 14 show, for SF-combs of $N_{\mathrm{F}}=6$, mean squared displacements and incoherent scattering functions of selected F-monomers. These are labeled as $\mathrm{F}_{n}$ with $n=1,2, \ldots, 6$ from the SF-link to the free end of the F-arm. At times after the decaging regime, an increasing dispersion is observed in the mobility of the F-monomers. For the highest investigated $T$, dispersion reaches a maximum at $t \approx 10^{3}$, and data for the different F-monomers approach a common limit at long times. A final merging is also expected for lower $T$, at times 


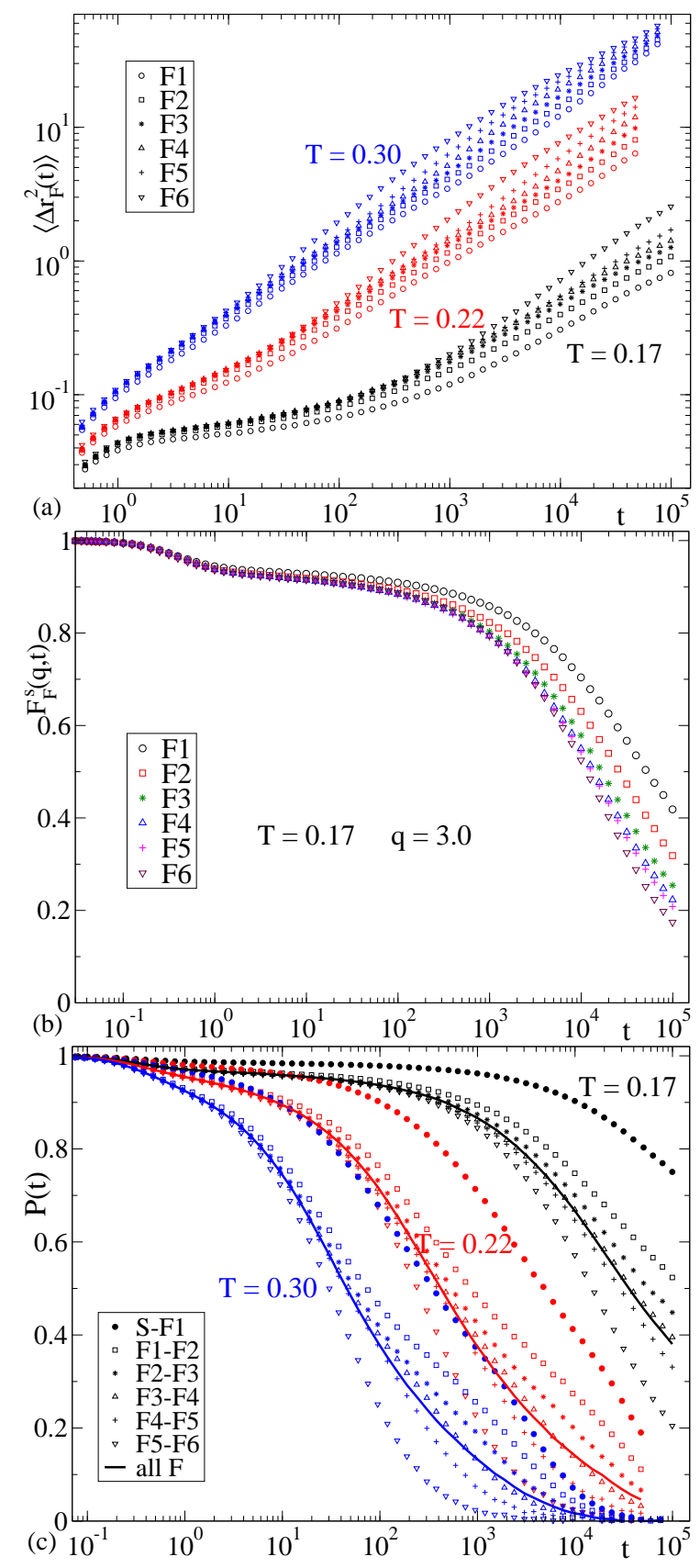

FIG. 14: For SF-combs of $N_{\mathrm{F}}=6$, dynamic correlators for specific locations (see text) of fast monomers $\left(\mathrm{F}_{n}\right)$ and fast bonds $\left(\mathrm{F}_{n}-\mathrm{F}_{n+1}\right)$. (a): $\mathrm{MSD}$ for $T=0.17$ (black), $T=0.22$ (red) and $T=0.30$ (blue). (b): incoherent scattering functions for $q=3.0$ and $T=0.17$. (c): orientational bond correlators (symbols) for $T=0.17$ (black), $T=0.22$ (red) and $T=0.30$ (blue). Also included as solid lines are the correlators averaged over all F-F bonds. In panels (a) and (c) data sets at different $T$ with a same symbol code correspond to a same specific location. 


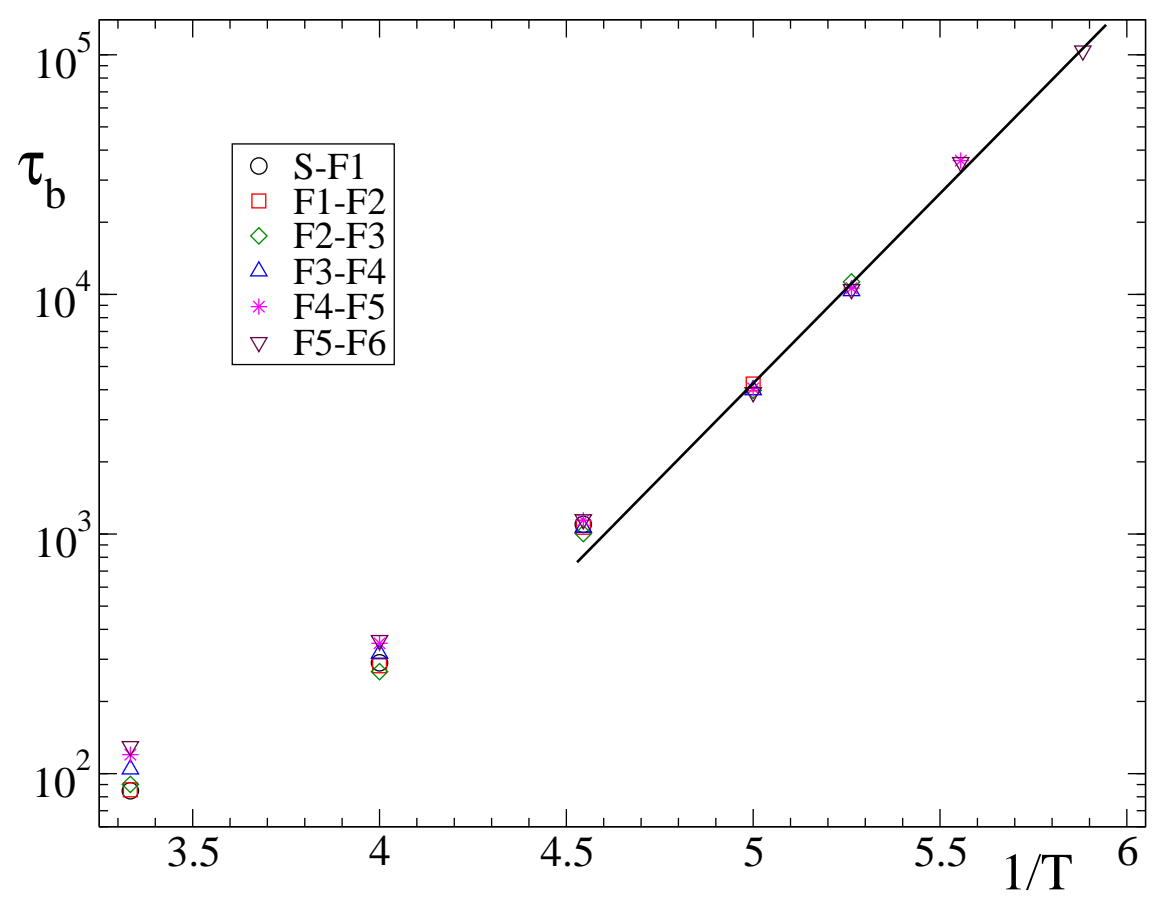

FIG. 15: For SF-combs of $N_{\mathrm{F}}=6$, temperature dependence of the reorientation time of specific bonds. Each set is rescaled by a factor $f$ in order to obtain data overlap ( $f=1$ for bonds $\mathrm{F}_{5}-\mathrm{F}_{6}$ ). The line indicates approximate Arrhenius behavior.

beyond the simulation window. At fixed temperature, the mobility of the F-monomers increases monotonically from the SF-link to the arm end. The observed gradients of mobility are however moderate at the investigated temperatures. Data in panels (a) and (b) show a dispersion of less than one decade in characteristic times. The experimental data for poly $(n-$ alkyl methacrylates) [21, 22] were also analyzed in terms of gradients of mobility along the arms. The phenomenological analysis provided distributions of relaxation times of up to seven decades for temperatures qualitatively equivalent to those investigated here, i.e., with equivalent time scales for the total $F(q, t)$. The authors of Refs. [21, 22] suggested that such a strong dispersion might be unrealistic and just an artifact of the assumed distribution. The weak dispersion observed in the simulations seems to confirm this guess.

Bond reorientation can be quantified by the correlator $P(t)=\langle\mathbf{b}(t) \cdot \mathbf{b}(0)\rangle /\left\langle b^{2}(0)\right\rangle$, with b the considered bond vector. Figure 14c shows, for SF-combs of $N_{\mathrm{F}}=6$, results for the orientational correlator of the S-F bond and the different F-F bonds. These are denoted as $\mathrm{F}_{n}-\mathrm{F}_{n+1}$, where the index $n$ is defined as for the data of panels (a) and (b). In analogy with the observations in such panels for translational dynamics, bond reorientation becomes 
faster as the arm end is approached. Reorientation for the $\mathrm{S}-\mathrm{F}$ bonds is significantly slower than for any of the F-F bonds. The dispersion in the reorientation times extends over about one decade, and does not seem to increase with decreasing temperature in the investigated range. This is suggested by results in Figure 15 for reorientation times $\tau_{\mathrm{b}}$ of the S-F bonds and all the different F-F bonds. We have estimated $\tau_{\mathrm{b}}$ by the criterium $P\left(\tau_{\mathrm{b}}\right)=0.2$. The temperature dependence of $\tau_{\mathrm{b}}$ is shown in Figure 15, where each data set corresponds to a specific bond. Moreover each set has been rescaled by factor in order to obtain data overlap. In analogy with the results of Figure 11, we use an Arrhenius representation. Almost perfect overlap is observed for $T \lesssim 0.22$, where data are compatible with Arrhenius behavior $\tau_{\mathrm{b}}=\tau_{\infty} \exp \left(E / \mathrm{k}_{\mathrm{B}} T\right)$, and with a very similar activation energy. According to this, the dispersion of about one decade in the reorientation times of Figure 14, is esentially due to a similar dispersion in the prefactors $\tau_{\infty}$ for the different bonds. These results may be relevant for the interpretation of experiments by dielectric spectroscopy, which can probe bond reorientation over much longer time scales at lower temperatures. If the extrapolation of the apparent Arrhenius behavior is correct, gradients of mobility at lower temperatures will not be stronger than those observed in Figure 14.

\section{CONCLUSIONS}

We have presented a computational investigation of a simple bead-spring model for selfassembled comb copolymers, where monomers in the main chain and in the arms are segregated in nanodomains. The observed structural and dynamic features have been discussed in comparison with recent findings in poly(n-alkyl methacrylates). The generic character of the bead-spring model suggests that the observed qualitative features are not specific of these systems. On the contrary, we propose that the former will be general features of self-assembled comb copolymers with dynamic asymmetry. These include a decoupling of self- and collective dynamics for the arm monomers in the nanodomain scale. The latter also exhibit strongly stretched relaxation. At least for time scales probed by neutron scattering experiments, stretching seems to be an intrinsic feature, not related to strong gradients of mobility along the arms. Finally, nanosegregation of main chains and arms can arise as a purely entropic effect, provided that the density of branch points is large enough. 


\section{ACKNOWLEDGEMENTS}

We acknowledge financial support from the projects FP7-PEOPLE-2007-1-1-ITN (DYNACOP, EU), MAT2007-63681 (Spain), and IT-436-07 (GV, Spain).

[1] Heijboer, J. In Physics of Non-Crystalline Solids; Prins, J.A., Ed.; North-Holland: Amsterdam, 1965; p 231.

[2] McCrum, N.G.; Read, B.E., Williams, G. Anelastic, Dielectric Effects in Polymeric Solids; Wiley: London, 1967.

[3] Cowie, J.M.G. J. Macromol. Sci.-Phys. B 1980, 18, 569.

[4] Meier, G.; Fytas, G., Dorfmüller, T. Macromolecules 1984, 17, 957.

[5] Giebel, L.; Meier, G.; Fytas, G.; Fischer, E. W. J. of Polym. Sci. B: Polym. Phys. 1992, 30, 1291.

[6] Garwe, F.; Schönhals, A.; Lockwenz, H.; Beiner, M.; Schröter, K.; Donth, E. Macromolecules $199629,247$.

[7] Schröter, K.; Unger, R.; Reissig, S.; Garwe, F.; Kahle, S.; Beiner, M.; Donth, E. Macromolecules 1998, 31, 8966 .

[8] Floudas, G.; Stepanek, P. Macromolecules 1998, 31, 6951.

[9] Beiner, M.; Schröter, K.; Hempel, E.; Reissig, S.; Donth, E. Macromolecules 1999,32, 6278.

[10] Dudognon, E.; Bernès, A.; Lacabanne, C. Macromolecules 2001, 34, 3988.

[11] Dudognon, E.; Bernès, A.; Lacabanne, C. Macromolecules 2002, 35, 5927.

[12] Beiner, M. Macromol. Rapid Commun. 2001,22, 869.

[13] Beiner, M.; Kabisch, O.; Reichl, S.; Huth, H. J. Non-Cryst. Sol. 2002, 307, 658.

[14] Hempel, E.; Beiner, M.; Huth, H.; Donth, E. Thermochimica Acta 2002, 391, 219.

[15] Pascui, O.; Beiner, M.; Reichert, D. Macromolecules 2003, 36, 3992.

[16] Beiner, M.; Huth H. Nature Mater. 2003, 2, 595.

[17] Hiller, S.; Pascui, O.; Kabisch, O.; Reichert, D.; Beiner, M. New Journal of Physics 2004, 6, 1.

[18] Menissez, C.; Sixou, B.; David, L.; Vigier G. J. Non-Cryst. Sol. 2005, 351, 595.

[19] Wind, M.; Graf, R.; Renker, S.; Spiess, H. W. Macromol. Chem. Phys. 2005, 206, 142. 
[20] Beiner, M. CP832, Flow Dynamics, The Second International Conference on Flow Dynamics, edited by M. Tokuyama and S. Maruyama, American Institute of Physics, 2006; p. 134.

[21] Arbe, A.; Genix, A.-C.; Colmenero, J.; Richter, D.; Fouquet, P. Soft Matter 2008, 4, 1792.

[22] Arbe, A.; Genix, A.-C.; Arrese-Igor, S.; Colmenero, J.; Richter, D. Macromolecules 2010, 43, 3107.

[23] Gaborieau, M.; Graf, R.; Kahle, S.; Pakula, T.; Spiess H.W. Macromolecules 2007, 40, 6249.

[24] Cowie, J.M.G.; Haq, Z.; McEwen, I.J.; Velickovic, J. Polymer 1981, 22, 327.

[25] Arrighi, V.; Triolo, A.; McEwen, I.J.; Holmes, P.; Triolo, R.; Amenitsch, H. Macromolecules 2000, 33, 4989.

[26] Genix, A.-C.; Lauprêtre, F. Macromolecules 2005, 38, 2786.

[27] Pankaj, S.; Hempel, E.; Beiner, M. Macromolecules 2009, 42, 716.

[28] Pankaj, S.; Beiner, M. J. Phys. Chem. B 2010, 114, 15459.

[29] McCreight, K.W.; Ge, J.J.; Guo, M.; Mann, I,; Li, F.; Shen, Z.; Jin, X.; Harris, F.W.; Cheng, S.Z.D. J. Polym. Sci.: Part B: Polym. Phys. 1999, 37, 1633.

[30] Kim, H.; Park, S.-B.; Jung, J.C.; Zin, W.-C. Polymer 1996, 37, 2845.

[31] Cervinka, L.; Ballauff, M. Colloid \& Polym. Sci. 1992, $270,859$.

[32] Kricheldorf, H.R.; Domschke, A. Macromolecules 1996, 29, 137.

[33] Laredo, E.; Grimau, M.; Bello, A.; López-Carrasquero, F. J. Non-Cryst. Sol. 2007, 353, 4324.

[34] Sanchis, M.J.; Carsí, M.; Ortiz-Serna, P., Domínguez-Espinosa, G.; Díaz-Calleja, R.; Riande, E.; Alegría, L.; Gargallo, L.; Radić, D. Macromolecules 2010, 43, 5723.

[35] Binder, K.; Baschnagel, J.; Paul, W. Prog. Polym. Sci. 2003, 28, 115.

[36] Baschnagel, J.; Varnik, F. J. Phys.: Condens. Matter 2005, 17, R851.

[37] Barrat, J.L.; Baschnagel, J.; Lyulin, A. Soft Matter 2010, 6, 3430.

[38] Weeks, J.D.; Chandler, D.; Andersen, H.C. J. Chem. Phys. 1971, 54, 5237.

[39] Kremer, K.; Grest, G.S. J. Chem. Phys. 1990, 92, 5057.

[40] Frenkel, D.; Smit, B. Understanding Molecular Simulation; Academic Press (Elsevier): San Diego, 1996.

[41] Martyna, G.J.; Tuckerman, M.E.; Tobias, D.J.; Klein, M.L. Mol. Phys. 1996, 87, 1117.

[42] Lovesey, S. W. Theory of Neutron Scattering from Condensed Matter; Clarendon Press: Oxford, 1984.

[43] For the orientational average of the wave vector $\mathbf{q}$, it must be noted that the differ- 
ent directions are constrained by the periodic boundary conditions of the box, i.e., $\mathbf{q}=$ $\left[2 \pi /\left\langle L_{\mathrm{box}}\right\rangle\right]\left(n_{x}, n_{y}, n_{z}\right)$, with $n_{\gamma}$ integer numbers and $\left\langle L_{\mathrm{box}}\right\rangle$ the box size averaged over the production NPT-run. The standard deviation of $L_{\text {box }}$ is smaller than $4 \times 10^{-4}\left\langle L_{\text {box }}\right\rangle$ in all cases, so that box size fluctuations have negligible effects in $q$-dependent correlators. In order to improve statistics for a given modulus $q_{0}$, we select all the sets $\left(n_{x}, n_{y}, n_{z}\right)$ yielding wave vectors of modulus $q_{0}(1-\zeta)<q<q_{0}(1+\zeta)$, with a small tolerance $\zeta>0$. We use $\zeta=10^{-2}$ for $q_{0}<60 \pi /\left\langle L_{\text {box }}\right\rangle$. Otherwise we use $\zeta=10^{-3}$.

[44] Poly(n-butylmethacrylates) with different molecular weights [17] show a change in the X-ray patterns (decrease of the intensity and shift to higher $q$-value of the position of the first peak) for oligomers with less than 10 monomers. However, the first peak indicative for nanodomain formation is present even in chains of only 6 monomers. The polymers here simulated have a relatively small degree of polymerization. Though a direct mapping between them and real polymers is not possible, the simulated chains are obviously long enough to develop the characteristic pattern attributed to nanophase segregation.

[45] Frick, B. ; Richter, D.; Ritter, Cl. Europhys. Lett. 1989, 9, 557.

[46] Hamad, E.Z. J. Chem. Phys. 1999, 111, 5599.

[47] Alsunaidi, A.; Abu-Sharkh, B.F. J. Chem. Phys. 2003, 119, 9894.

[48] Richter, D.; Monkenbusch, M.; Arbe, A.; Colmenero, J. Neutron Spin Echo in Polymer Systems, edited by Advances in Polymer Science (Springer Verlag, Berlin Heidelberg New York, 2005) Vol. 174.

[49] Bernabei, M.; Moreno, A.J.; Colmenero, J. Phys. Rev. Lett. 2008, 101, 255701.

[50] Moreno, A.J.; Colmenero, J. J. Chem. Phys 2006, 124, 184906.

[51] Brodeck, M.; Alvarez, F.; Moreno, A.J.; Colmenero, J.; Richter, D. Macromolecules 2010, 43, 3036.

[52] Busselez, R.; Arbe, A.; Alvarez, F.; Colmenero, J.; Frick, B. (submitted).

[53] Doi, M.; Edwards, S. F. The Theory of Polymer Dynamics, Oxford University Press: Oxford, 1986.

[54] Moreno, A.J.; Colmenero, J. Phys. Rev. E 2006, 74, 021409.

[55] Höfling, F.; Franosch, T.; Frey, E. Phys. Rev. Lett. 2006, 96, 165901.

[56] Kurzidim, J.; Coslovich, D.; Kahl, G. Phys. Rev. Lett. 2009, 103, 138303.

[57] Kim, K.; Miyazaki, K.; Saito, S. EPL 2009, 88, 36002. 
[58] Voigtmann, Th.; Horbach, J. Phys. Rev. Lett. 2009, 103, 205901.

[59] Moreno, A.J.; Colmenero, J. J. Chem. Phys 2006, 125, 164507.

[60] Voigtmann, Th. arXiv:1010.0440.

[61] Moreno, A.J.; Colmenero, J. J. Phys.: Condens. Matter 2007, 19, 466112.

[62] Mayer, C.; Sciortino, F.; Likos, C.N.; Tartaglia, P.; Löwen, H.; Zaccarelli, E. Macromolecules 2009, 42, 423 .

[63] Voigtmann, Th.; Horbach, J. Europhys. Lett. 2006, 74, 459.

[64] Jund, P.; Kob, W.; Jullien, R. Phys. Rev. B 2002, 64, 134303.

[65] Farago, B.; Arbe, A.; Colmenero, J.; Faust, R.; Buchenau, U.; Richter, D. Phys. Rev. E 2002, $65,051803$.

[66] Donati, C.; Glotzer, S.C.; Poole, P.H.; Kob, W.; Plimpton, S.J. Phys. Rev. E 1999, 60, 3107.

[67] Kob, W.; Donati, C.; Plimpton, S.J.; Poole, P.H.; Glotzer, S.C. Phys. Rev. Lett. 1997, 79, 2827. 

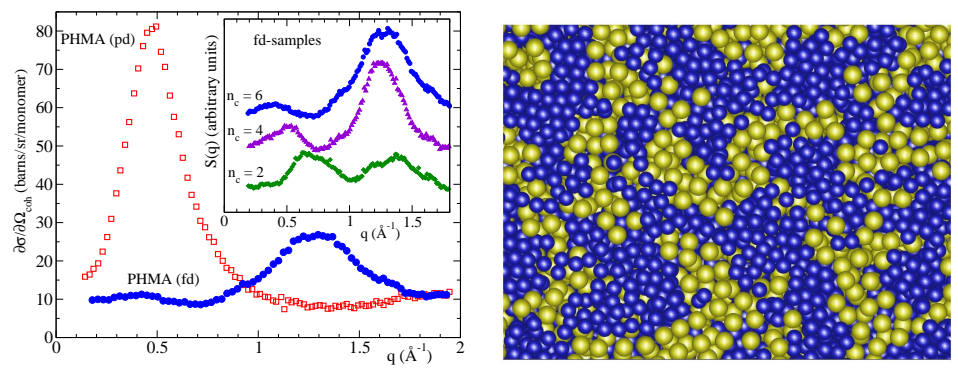

\section{TABLE OF CONTENTS GRAPHIC}

Structure and Dynamics of Self-assembled Comb Copolymers: Comparison between Simulations of a Generic Model and Neutron Scattering Experiments

Angel J. Moreno, Arantxa Arbe and Juan Colmenero 